\title{
Existence for an Allen-Cahn/Cahn-Hilliard system with degenerate mobility
}

\author{
ROBERTA DAL PASSO ${ }^{\dagger}$ \\ Dipartimento di Matematica, Università di Roma “Tor Vergata”, 00133 Roma, Italy \\ LORENZO GIACOMELLI ${ }^{\ddagger}$ \\ Dipartimento di Matematica, Università di Roma "La Sapienza”, 00185 Roma, Italy
}

AND

AMY NOVICK-COHEN ${ }^{\S}$

Department of Mathematics, Technion-IIT, Haifa, Israel 32000

[Received 30 September 1998 and in revised form 10 May 1999]

\begin{abstract}
We prove existence in one space dimension of weak solutions for the Neumann problem for a degenerate parabolic system consisting of a fourth-order and a second-order equation with singular lower-order terms. This system arises in the description of phase separation and ordering in binary alloys.
\end{abstract}

\section{Introduction}

Our paper is concerned with the Allen-Cahn/Cahn-Hilliard system

$$
(\mathbf{P}) \begin{cases}u_{t}=\left[\omega_{1} Q(u, v)\left(\mathcal{F}_{u}(u, v)-\varepsilon u_{x x}\right)_{x}\right]_{x} & (x, t) \in \Omega_{T} \\ v_{t}=-\omega_{2} Q(u, v)\left(\mathcal{F}_{v}(u, v)-\varepsilon v_{x x}\right) & (x, t) \in \Omega_{T} \\ u_{x}=v_{x}=\omega_{1} Q(u, v)\left(\mathcal{F}_{u}(u, v)-\varepsilon u_{x x}\right)_{x}=0 & (x, t) \in S_{T} \\ u(x, 0)=u_{0}(x), \quad v(x, 0)=v_{0}(x) & x \in \Omega \\ (u, v) \in \bar{B} & (x, t) \in \Omega_{T}\end{cases}
$$

where $\Omega \subset \mathbb{R}$ is a bounded open interval, $T>0, \Omega_{T}=\Omega \times(0, T)$ and $S_{T}=\partial \Omega \times(0, T)$. The dimensionless homogeneous free energy $\mathcal{F}$ is assumed here to have the form

$$
\mathcal{F}(u, v)=F(u+v)+F(u-v)+F(1-(u+v))+F(1-(u-v))+\alpha u(1-u)-\beta v^{2},
$$

where $F(s)=\frac{\Theta}{2} s \ln s$ and $\Theta$ denotes the (dimensionless) absolute temperature; the function $\mathcal{F}$ is defined in the square

$$
B=\left\{(u, v) \in \mathbb{R}^{2}: 0<u+v<1,0<u-v<1\right\},
$$

and $Q(u, v)$, the mobility, is non-negative. Here $\varepsilon$ is a gradient energy coefficient, and $\varepsilon, \alpha, \beta$, $\omega_{1}$ and $\omega_{2}$ are positive constants. The system $(\mathbf{P})$ with constant mobility was introduced by Cahn

\footnotetext{
†Email: dalpasso@mat.uniroma2.it

†Email: giacomel@mat.uniroma1.it

§Email: amync@techunix.technion.ac.il
} 
\& Novick-Cohen [13] in the description of simultaneous phase separation and order-disorder transition in a BCC Fe-Al binary alloy: $u$ and $v$ represent a conserved (typically an average concentration) and a non-conserved order parameter, respectively. See [13] for further relevant references. In the context of their derivation $\omega_{1}$ and $\omega_{2}$ should assume the values $4 \varepsilon^{2}$ and $\frac{1}{4}$ respectively. For the sake of simplicity in our analysis we let $\omega_{1}=\omega_{2}=1$, though our conclusions are valid for arbitrary positive values of $\omega_{1}$ and $\omega_{2}$. The derivation in [13] can be extended to allow a non-constant mobility, and in the present paper we shall make the further assumption that the mobility $Q$ is given by

$$
Q(u, v)=u(1-u)\left(\frac{1}{4}-v^{2}\right)=: Q_{1}(u) Q_{2}(v) ;
$$

such a form satisfies the physical notion that the mobility should vanish at the pure phases and also turns out to be analytically convenient to consider.

It is known that Allen-Cahn and Cahn-Hilliard equations can serve as diffuse interface models for limiting sharp interface motion. The Allen-Cahn equation serves as a diffuse interface model for antiphase grain boundary coarsening in the sense that a singular limit of the equation ([1],[28],[22]) yields a geometric problem in which a sharp interface separating two phase variants evolves according to motion by mean curvature $(V=M \kappa)$. The Cahn-Hilliard equation was constructed to describe mass conservative phase separation, and by considering an appropriate singular limit can describe the motion of interphase boundaries separating two phases of differing composition during the later stages of coarsening. For the constant mobility case with a non-singular free energy, this limiting motion ([27], [16]) is given by a free boundary problem known as a Mullins-Sekerka problem. For the Cahn-Hilliard equation with a degenerate mobility and with a free energy with logarithmic terms (as in (P) setting $v=0$ ), it has been shown [12] that when $\Theta=\mathcal{O}\left(\varepsilon^{1 / 2}\right)$, the limiting geometric boundary motion is predicted to be given by $V=-M \triangle_{s} \kappa$; i.e. surface diffusion. Rigorous justification has yet to be given for the surface diffusion description; however, the numerical results of Blowey \& Elliott [8], Barrett \& Blowey [2], and Barrett, Blowey, \& Garcke [3] lend credibility to these results. Similarly, a singular limit for a system of Allen-Cahn equations can yield geometric motion in which an array of antiphase boundaries move by motion by mean curvature and couple together at triple junctions according to Young's law [11], and singular limits for a system of Cahn-Hilliard equations can lead to an array of interphase boundaries governed by a generalized Mullins-Sekerka problem [10] or by motion by minus the surface Laplacian [23].

As remarked above, by its construction, the Allen-Cahn/Cahn-Hilliard system was designed to describe simultaneous order-disorder and phase separation. By examining the free energy prescribed in Problem $(\mathbf{P})$, it is clear that at low temperatures three phases - a disordered phase given by $(u, v) \approx(0,0)$ or $(1,0)$, and two ordered phase variants given by $(u, v) \approx\left(\frac{1}{2}, \pm \frac{1}{2}\right)-$ should roughly dominate the system at late times when it is nearly equilibrated. A description of the motion of both antiphase boundaries (APBs) separating ordered phase variants and interphase boundaries (IPBs) separating ordered variants from regions of disordered phase should be provided by the Allen-Cahn/Cahn-Hilliard system $(\mathbf{P})$ in an appropriate singular limit. In the proximity of the deep quench limit $\left(\mathcal{O}\left(\varepsilon^{1 / 2}\right)\right)$, formal asymptotic analysis for Problem $(\mathbf{P})$ (Cahn \& NovickCohen [14], Novick-Cohen [25]) has predicted geometric motion in which APBs are governed by motion by mean curvature, IPBs move by motion by minus the surface Laplacian of the curvature, and there is quasi-static diffusion of the chemical potential along APBs. Under the assumption that the curvature of APBs is $\mathcal{O}\left(\varepsilon^{-1 / 2}\right)$ and the curvature of IPBs is $\mathcal{O}\left(\varepsilon^{3 / 2}\right)$, the two motions occur on the same time scale, and as in the analysis for a system of degenerate Cahn-Hilliard equations 
[23], triple junction motion is governed by balance of mass flux, Young's law, and continuity of the chemical potential. To leading order, complete wetting is predicted; i.e. two IPBs connecting up with an APB at a triple junction meet with an angle of zero between them. However, the previously mentioned analysis allows the possibility of dynamic inhibition of complete wetting; i.e. the angle between two IPBs meeting at a triple junction may be small but non-vanishing. We remark that recently, Cahn \& Novick-Cohen [15] studied limiting motion for an Allen-Cahn/CahnHilliard system with non-constant mobility and a polynomial free energy designed to model the neighborhood of a tricritical point. By varying the values of the free energy polynomial parameters, they were able to mimic various levels of adsorption, prewetting, and impurity drag. Also, as discussed in [25], the Allen-Cahn/Cahn-Hilliard system can be viewed as a diffuse interface model for sintering of small grains and grain boundary grooving of polycrystalline films.

The analytical study of the Cahn-Hilliard equation in the case of constant mobility and logarithmic free energy is due to Elliott \& Luckhaus [21] who demonstrated existence and uniqueness. By passing to the limit $\Theta \rightarrow 0$, they obtained existence and uniqueness also in the deep quench limit; i.e. with a free energy of the form

$$
\mathcal{F}(u)= \begin{cases}u(1-u) & 0 \leqslant u \leqslant 1 \\ +\infty & \text { elsewhere, }\end{cases}
$$

as had been proven earlier by Blowey \& Elliott [7]. The Cahn-Hilliard equation with non-singular lower-order terms was considered by Novick-Cohen in [26], where the effect of a concentration dependent non-degenerate mobility on stability was studied. The degenerate case with possibly singular free energy was studied by Elliott \& Garcke [19] who proved existence under general assumptions on $Q$ and $\mathcal{F}$ which include the case

$$
\begin{gathered}
Q(u)=u(1-u), \\
\mathcal{F}(u)=F(u)+F(1-u)+\alpha u(1-u) ;
\end{gathered}
$$

existence for non-degenerate mobilities with a possibly singular free energy is a byproduct of [19]. In the case of concentration dependent non-degenerate mobilities with a logarithmic free energy, uniqueness was proven by Barrett \& Blowey [2] for sufficiently regular initial data. Recently, Elliott \& Garcke [20] and Garcke \& Novick-Cohen [23] have obtained existence results for respectively systems of Cahn-Hilliard equations with degenerate mobilities and the limiting geometric motions to which such systems give raise in the long time, low temperature limit. We mention that systems of Allen-Cahn/Cahn-Hilliard type with non-singular lower-order terms and non-degenerate mobilities have been considered by Brochet, Hilhorst, \& Novick-Cohen in [9], where global existence as well as existence of a global attractor and inertial sets was proven.

The problem of existence of solutions for system $(\mathbf{P})$ is delicate in several respects. In fact, this kind of operator combines together various non-trivial features. For example, it is known (see [4], [17], [18], [6]) that the degeneracy of the principal part leads, even when considering each equation in the system separately, several open problems on well-posedness. Below we shall describe some of the problematics in detail (see also Remarks 1.2 and 1.3).

In considering Problem $(\mathbf{P})$, one has to deal with the constraint $(u, v) \in \bar{B}$; in addition, note that the partial derivatives of $\mathcal{F}$ can be extended to be continuous functions with values in $\mathbb{R} \cup\{ \pm \infty\}$ only in $\bar{B} \backslash\left\{(0,0),(1,0),\left(\frac{1}{2}, \pm \frac{1}{2}\right)\right\}$, and not in $\bar{B}$. This difficulty can be handled in the case of 
positive mobilities via a-priori estimates in $L^{1}\left(\Omega_{T}\right)$ on the terms

$$
F^{\prime}(u+v), \ldots, F^{\prime}(1-(u-v))
$$

which are logarithmic. This estimate implies that $(u, v) \in B$ almost everywhere in $\Omega_{T}$ in the case of positive mobilities. Another peculiarity in the structure of the operator, which turns out to be crucial in our analysis, is the fact that the mobility degenerates only at the vertices of the box $B$, whereas the derivatives of $\mathcal{F}$ are singular over the whole boundary $\partial B$. Therefore there is no obvious way to bound a-priori the product of mobilities and derivatives of $\mathcal{F}$. For a single degenerate $\mathrm{C}-\mathrm{H}$ equation such difficulties do not arise as the locus of the zeroes of the mobility and the singularities of the logarithmic terms appearing in the free energy is identical. Thus, this represents a main difference between the system which we consider here and the case of a single degenerate C-H equation [19]. On the other hand, the assumed form of $Q$ allows the derivation of an estimate for $v$, namely

$$
\int_{\Omega} \frac{1}{\left(\frac{1}{4}-v^{2}(t)\right)^{\beta-1}} \leqslant c+\int_{\Omega} \frac{1}{\left(\frac{1}{4}-v_{0}^{2}\right)^{\beta-1}} \quad \text { if } \beta \in(1,4] .
$$

This estimate permits control of the degeneracy of the system with respect to the variable $v$. It is worth noting that the estimate (1.2) only holds if one knows a-priori that the range of the solution is contained in the closure of the square. For this reason we first prove an existence result for the case of non-degenerate mobilities with a logarithmic free energy, whose solutions can be shown to satisfy $(u, v) \in \bar{B}$ by virtue of the estimates on terms of the type given in (1.1). Afterwards we derive an estimate in $L^{1}\left(\Omega_{T}\right)$ for the terms

$$
u(1-u) v F^{\prime}(u+v), \ldots, u(1-u) v F^{\prime}(1-(u-v))
$$

which holds for arbitrarily positive mobilities and implies that the constraint $(u, v) \in B \cup$ $\{(0,0),(1,0)\}$ is satisfied almost everywhere. Indeed, we are not able to exclude the possibility that the sets in $\Omega_{T}$ where $(u, v)=(0,0)$ or $(u, v)=(1,0)$ have positive measure -a possibility which has also not been excluded in the case of a single degenerate $\mathrm{C}-\mathrm{H}$ equation with free energy and mobility of analogous form.

Throughout the paper we make the following assumption on the initial data:

$$
\begin{gathered}
u_{0} \in H^{1}(\Omega), \quad v_{0} \in H^{1}(\Omega), \\
\left(u_{0}, v_{0}\right) \in \bar{B}, \quad v_{0} \in(-1 / 2,1 / 2), \quad \overline{u_{0}} \in(0,1),
\end{gathered}
$$

where $\bar{f}$ denotes the mean value of $f$ in $\Omega$ of a given $f \in L^{1}(\Omega)$. Let us introduce some notation which we shall use in the sequel. Given $u, v \in C\left(\bar{\Omega}_{T}\right)$, we define the sets

$$
\begin{gathered}
\mathcal{D}_{\eta}(u)=\left\{(x, t) \in \bar{\Omega}_{T}: \eta<u(x, t)<1-\eta\right\}, \quad 0 \leqslant \eta<\frac{1}{2}, \\
\mathcal{B}(u, v)=\left\{(x, t) \in \bar{\Omega}_{T}:(u(x, t), v(x, t)) \in B\right\},
\end{gathered}
$$

with the convention that $\mathcal{D}(u)=\mathcal{D}_{0}(u)$. We denote by $\langle\cdot, \cdot\rangle$ the duality pairing between $\left(H^{1}(\Omega)\right)^{\prime}$ and $H^{1}(\Omega)$.

Let us define our concept of a weak solution for Problem $(\mathbf{P})$ as follows.

Definition 1 A triplet $(u, v, w)$ is called a weak solution of $(\mathbf{P})$ if: 
(i) $u \in C^{0, \frac{1}{2}, \frac{1}{8}}\left(\bar{\Omega}_{T}\right) \cap L^{\infty}\left(0, T ; H^{1}(\Omega)\right)$, $v \in C^{0, \frac{1}{2}, \frac{1}{4}}\left(\bar{\Omega}_{T}\right) \cap L^{\infty}\left(0, T ; H^{1}(\Omega)\right)$, $w \in L^{2}\left(\Omega_{T}\right)$;

(ii) $u_{x x} \in L_{\mathrm{loc}}^{2}(\mathcal{D}(u)), v_{x x} \in L_{\mathrm{loc}}^{2}(\mathcal{D}(u))$;

(iii) $u_{t} \in L^{2}\left(0, T ;\left(H^{1}(\Omega)\right)^{\prime}\right), v_{t} \in L^{2}\left(\Omega_{T}\right)$;

(iv) $(u, v) \in \bar{B}, v \in(-1 / 2,1 / 2)$;

(v) $\mathcal{F}_{u}(u, v) \in L_{\text {loc }}^{2}(\mathcal{D}(u)), \mathcal{F}_{v}(u, v) \in L_{\text {loc }}^{2}(\mathcal{D}(u))$;

(vi) $u(x, 0)=u_{0}(x), v(x, 0)=v_{0}(x)$;

(vii) $\left.u_{x}\right|_{S_{T} \cap \mathcal{D}(u)}=\left.v_{x}\right|_{S_{T} \cap \mathcal{D}(u)}=0$ in $L_{\text {loc }}^{2}\left(S_{T} \cap \mathcal{D}(u)\right)$;

(viii) $(u, v, w)$ solves the system $(\mathbf{P})$ in the following sense:

$$
\begin{gathered}
\int_{0}^{T}\left\langle u_{t}, \phi\right\rangle \mathrm{d} t=-\iint_{\Omega_{T}}[Q(u, v)]^{1 / 2} w \phi_{x} \quad \forall \phi \in L^{2}\left(0, T ; H^{1}(\Omega)\right), \\
\iint_{\Omega_{T}} v_{t} \psi=-\iint_{\Omega_{T}} Q(u, v)\left(\mathcal{F}_{v}(u, v)-\varepsilon v_{x x}\right) \psi \quad \forall \psi \in L^{2}\left(\Omega_{T}\right): \operatorname{supp} \psi \subset \mathcal{D}(u),
\end{gathered}
$$

where $w=Q^{1 / 2}(u, v)\left(\mathcal{F}_{u}(u, v)-\varepsilon u_{x x}\right)_{x}$ in the sense that

$$
\iint_{\Omega_{T}} w \phi=-\iint_{\Omega_{T}}\left(\mathcal{F}_{u}(u, v)-\varepsilon u_{x x}\right)\left([Q(u, v)]^{1 / 2} \phi\right)_{x}
$$

for all $\phi \in L^{2}\left(0, T ; H_{0}^{1}(\Omega)\right)$ with $\operatorname{supp} \phi \subset \mathcal{D}(u)$.

REMARK 1.1 The terms in the Definition are all well defined. In particular:

1. The traces of $u_{x}, v_{x}$ in $S_{T} \cap \mathcal{D}(u)$ can be seen to be well defined by the following argument: Let $K \subset S_{T} \cap \mathcal{D}(u)$ compact; then $K \subseteq S_{T} \cap \mathcal{D}_{\eta}(u)$ for some $\eta>0$. Since $u \in C\left(\bar{\Omega}_{T}\right)$, we can select $\zeta \in C^{\infty}\left(\bar{\Omega}_{T}\right)$ such that $0 \leqslant \zeta \leqslant 1, \zeta=1$ in $\mathcal{D}_{\eta}(u)$ and $\zeta=0$ in $\bar{\Omega}_{T} \backslash \mathcal{D}_{\eta / 2}(u)$. Then $\zeta u \in L^{2}\left(0, T ; H^{2}(\Omega)\right)$ and therefore $\left.(\zeta u)_{x}\right|_{S_{T}} \in L^{2}\left(0, T ; L^{2}(\partial \Omega)\right)$. It follows that

$$
\left.u_{x}\right|_{S_{T} \cap K} \in L^{2}\left(S_{T} \cap K\right) .
$$

The argument for $v_{x}$ is the same.

2. Since (iv) implies that $Q_{2}(v)$ is strictly positive, from (i) it then follows that the function $\left([Q(u, v)]^{1 / 2} \phi\right)_{x}$ has compact support in $\mathcal{D}(u)$ and belongs to $L^{2}\left(\Omega_{T}\right)$ for any $\phi \in$ $L^{2}\left(0, T ; H^{1}(\Omega)\right)$ with supp $\phi \subset \mathcal{D}(u)$. Therefore the integral at the right hand side of (1.7) makes sense.

REMARK 1.2 Comparison with other definitions of solution.

The choice of test functions which are compactly supported in $\mathcal{D}(u)$, as well as the regularity properties given by $(i i)$ and $(v)$, differ from (and actually weaken) the definition of solution given in [19] in the case of a single degenerate $\mathrm{C}-\mathrm{H}$ equation. This reflects the lack of control on the set 
$\{u=0,1\}$, which is essentially due to the difference between the locus of the zeroes of $Q$ and that of the singularities of $\mathcal{F}_{u}, \mathcal{F}_{v}$.

Note that our choice of the unknowns - which were taken in [19] to be $(u, v, J)$ with $J=$ $Q(u, v)\left(\mathcal{F}_{u}-\varepsilon u_{x x}\right)_{x}$-is not weaker: indeed, choosing $\phi=\sqrt{Q} \varphi$ in (1.7), one can identify the flux $J$ in the sense that

$$
\int_{0}^{T}\left\langle u_{t}, \phi\right\rangle \mathrm{d} t=-\iint_{\Omega_{T}} J \phi_{x} \quad \forall \phi \in L^{2}\left(0, T ; H^{1}(\Omega)\right),
$$

where

$$
\iint_{\Omega_{T}} J \varphi=-\iint_{\Omega_{T}}\left(\mathcal{F}_{u}-\varepsilon u_{x x}\right)(Q \varphi)_{x}
$$

for all $\varphi \in L^{2}\left(0, T ; H_{0}^{1}(\Omega)\right)$ with $\operatorname{supp} \varphi \subset \mathcal{D}(u)$.

\section{REMARK 1.3 Uniqueness.}

According to our definition, problem (P) admits more than one solution. To demonstrate this, let us take $v \equiv 0$; then $(\mathbf{P})$ formally reduces to a single $\mathrm{C}-\mathrm{H}$ equation to be solved in $\mathcal{D}(u)$. Hence, this is a free boundary problem (the free boundary being given by $\partial \mathcal{D}(u)$ ), but our definition does not specify any free boundary condition. Note that the definition of solution given in [19], and in particular the regularity property $u \in L^{2}\left(0, T ; H^{2}(\Omega)\right)$, does contain in one space dimension the free boundary condition: $\left.u_{x}\right|_{\partial \mathcal{D}(u)}=0$ almost everywhere. On this basis, it is strongly believed that solutions with this property should be unique; in fact, this is probably the major open problem in the present theory of fourth-order degenerate parabolic equations with principal part of the form $-\operatorname{div}(m(u) \nabla \Delta u), m \geqslant 0$.

We can actually construct an example of non-uniqueness according to our definition. Let $u(x)$ be the unique solution of the initial value problem

$$
\left\{\begin{array}{l}
\varepsilon u^{\prime \prime}=2 \log \frac{u}{1-u}+\alpha(1-2 u), \quad x>0 \\
u(0)=\bar{u}_{0}>0 \\
u^{\prime}(0)=0 .
\end{array}\right.
$$

It is easy to verify that for $\bar{u}_{0}=\bar{u}_{0}(\varepsilon, \alpha)$ sufficiently small there exists $x_{0}>0$ such that $u(x) \rightarrow 0$ as $x \nearrow x_{0}$; in addition

$$
u^{\prime}\left(x_{0}\right)=-c<0 .
$$

Define

$$
u_{0}(x)= \begin{cases}u(|x|) & |x|<x_{0} \\ 0 & \text { elsewhere }\end{cases}
$$

The pair $\left(u_{0}, 0\right)$ is, according to Definition 1, a steady solution of Problem $(\mathbf{P})$ in $\Omega=\left(-2 x_{0}, 2 x_{0}\right)$. Note that $u_{0}$ does not belong to $H^{2}\left(\left(-2 x_{0}, 2 x_{0}\right)\right)$. On the other hand, let $\tilde{u}$ denote a solution of the $\mathrm{C}-\mathrm{H}$ equation in the sense of [19] with initial data $u_{0}$. The pair $(\tilde{u}, 0)$ is also a solution of $(\mathbf{P})$ in the sense of Definition 1, and, since $\tilde{u} \in L^{2}\left(0, T ; H^{2}(\Omega)\right)$, we conclude that $\tilde{u}(t) \neq u_{0}$.

The non-degeneracy of the system with respect to the unknown $v$ given by (iv) should be required, as far as $v$ is concerned, to characterize uniquely a solution. This would not be the case if 
degeneracy with respect to $v$ was allowed: indeed, Dal Passo \& Luckhaus have proved ([18], see in particular Remark 1 and Section 3) that the Dirichlet problem for

$$
z_{t}=z \Delta z-z \ln z
$$

admits more than one solution for a suitable class of initial data.

The main result of the paper is the following

THEOREM 1 For all $u_{0}, v_{0}$ satisfying (1.4) there exists a weak solution of Problem (P) in the sense of Definition 1; in addition, the set

$$
\mathcal{D}(u) \backslash \mathcal{B}(u, v)=\left\{(x, t) \in \bar{\Omega}_{T}:(u(x, t), v(x, t)) \in \partial B \backslash\{(0,0),(1,0)\}\right\}
$$

has measure zero.

In Section 2 we obtain (by means of a Galerkin approximation) an existence result for suitably regularized systems; i.e. for systems with the form of Problem $(\mathbf{P})$ but which have been made to be uniformly parabolic by assuming sufficient additional regularity properties for $Q$ and $\mathcal{F}$. The proof of Theorem 1 is contained in Section 3, where by rescaling, without loss of generality we have assumed $\Theta=2$ : in Subsection 3.1 we define a two-parameter family of approximating regular systems and derive an energy inequality yielding uniform estimates for the corresponding solutions; in Subsection 3.2 we obtain intermediate results for non-degenerate systems based on a free energy which is now of the original $\operatorname{logarithmic}$ form, $\mathcal{F}(u, v)$; in Subsection 3.3 we use these approximating solutions to construct a weak solution of Problem $(\mathbf{P})$.

We observe that most of the results contained in Subsections 3.1 and 3.2 remain valid in higher space dimensions. Actually it is the continuity of the solution that enables us to control the boundary of the box $B$ uniformly with respect to the approximating procedure; however, the need for continuity forces our results to be restricted, up to now, to space dimension one.

\section{Regularized systems}

In this section we prove an existence theorem for the Allen-Cahn/Cahn-Hilliard system under additional regularity and positivity assumptions on the mobility and the homogeneous free energy. This result will be used later to construct approximating solutions to Problem (P).

Consider the following system:

$$
\left(\mathbf{P}^{\prime}\right) \begin{cases}u_{t}=\left[q_{1}(u, v)\left(f_{1}(u, v)-\varepsilon u_{x x}\right)_{x}\right]_{x} & (x, t) \in \Omega_{T} \\ v_{t}=-q_{2}(u, v)\left(f_{2}(u, v)-\varepsilon v_{x x}\right) & (x, t) \in \Omega_{T} \\ v_{x}=u_{x}=u_{x x x}=0 & (x, t) \in S_{T} \\ u(x, 0)=u_{0}(x), \quad v(x, 0)=v_{0}(x) & x \in \Omega\end{cases}
$$

where $q_{i}, f_{i}$ satisfy:

(H1) $q_{i} \in C\left(\mathbb{R}^{2} ; \mathbb{R}^{+}\right)$, with $q_{\min } \leqslant q_{i} \leqslant q_{\max }$ for some $0<q_{\min } \leqslant q_{\max }$;

(H2) $f_{1} \in C^{1}\left(\mathbb{R}^{2} ; \mathbb{R}\right)$ and $f_{2} \in C\left(\mathbb{R}^{2}, \mathbb{R}\right)$, with $\left\|f_{1}\right\|_{C^{1}}+\left\|f_{2}\right\|_{C^{0}} \leqslant F_{0}$ for some $F_{0}>0$. 
THEOREM 2.1 Assuming (H1), (H2) and $u_{0}, v_{0} \in H^{1}(\Omega)$, there exists a pair of functions $(u, v)$ such that:

(i) $u \in L^{\infty}\left(0, T ; H^{1}(\Omega)\right) \cap L^{2}\left(0, T ; H^{3}(\Omega)\right) \cap C\left([0, T] ; H^{\lambda}(\Omega)\right), \lambda<1$;

(ii) $v \in L^{\infty}\left(0, T ; H^{1}(\Omega)\right) \cap L^{2}\left(0, T ; H^{2}(\Omega)\right) \cap C\left([0, T] ; H^{\lambda}(\Omega)\right), \lambda<1$;

(iii) $u_{t} \in L^{2}\left(0, T ;\left(H^{1}(\Omega)\right)^{\prime}\right), v_{t} \in L^{2}\left(\Omega_{T}\right)$;

(iv) $u(0)=u_{0}$ and $v(0)=v_{0}$ in $L^{2}(\Omega)$;

(v) $\left.u_{x}\right|_{S_{T}}=\left.v_{x}\right|_{S_{T}}=0$ in $L^{2}\left(S_{T}\right)$;

(vi) $(u, v)$ solves $\left(\mathbf{P}^{\prime}\right)$ in the following sense:

$$
\begin{gathered}
\int_{0}^{T}\left\langle u_{t}, \phi\right\rangle \mathrm{d} t=-\iint_{\Omega_{T}} q_{1}(u, v)\left(f_{1}(u, v)-\varepsilon u_{x x}\right)_{x} \phi_{x} \quad \forall \phi \in L^{2}\left(0, T ; H^{1}(\Omega)\right) \\
\iint_{\Omega_{T}} v_{t} \psi=-\iint_{\Omega_{T}} q_{2}(u, v)\left(f_{2}(u, v)-\varepsilon v_{x x}\right) \psi \quad \forall \psi \in L^{2}\left(\Omega_{T}\right) .
\end{gathered}
$$

Proof. We apply a Galerkin approximation. Let us denote by $\psi_{i}, i \in \mathbf{N}$, the eigenfunctions of minus the Laplacian with Neumann boundary conditions:

$$
\begin{cases}-\psi_{i}^{\prime \prime}=\lambda_{i} \psi_{i} & x \in \Omega \\ \psi_{i}^{\prime}=0 & x \in \partial \Omega .\end{cases}
$$

Without loss of generality, we assume that the eigenfunctions $\psi_{i}$ are orthogonal in $H^{1}(\Omega)$, orthonormal in $L^{2}(\Omega)$, and that the first eigenvalue is zero $\left(0=\lambda_{1}<\lambda_{2} \ldots\right)$. The Galerkin ansatz for $\left(\mathbf{P}^{\prime}\right)$

$$
u^{N}(x, t)=\sum_{i=1}^{N} a_{i}^{N}(t) \psi_{i}(x), \quad v^{N}(x, t)=\sum_{i=1}^{N} b_{i}^{N}(t) \psi_{i}(x)
$$

yields the following initial value problem:

$$
\begin{aligned}
& \frac{\mathrm{d} a_{j}^{N}}{\mathrm{~d} t}=\int_{\Omega} u_{t}^{N} \psi_{j}=-\int_{\Omega} q_{1}\left(u^{N}, v^{N}\right)\left(f_{1}\left(u^{N}, v^{N}\right)-\varepsilon u_{x x}^{N}\right)_{x} \psi_{j x} \\
& \frac{\mathrm{d} b_{j}^{N}}{\mathrm{~d} t}=\int_{\Omega} v_{t}^{N} \psi_{j}=-\int_{\Omega} q_{2}\left(u^{N}, v^{N}\right)\left(f_{2}\left(u^{N}, v^{N}\right)-\varepsilon v_{x x}^{N}\right) \psi_{j} \quad j=1, \ldots, N \\
& a_{j}^{N}(0)=\left(u_{0}, \psi_{j}\right)_{L^{2}(\Omega)}, \quad b_{j}^{N}(0)=\left(v_{0}, \psi_{j}\right)_{L^{2}(\Omega)} .
\end{aligned}
$$

The problem has a local solution because the right hand side is continuous with respect to $\left(a_{1}^{N}, \ldots, a_{N}^{N}, b_{1}^{N}, \ldots, b_{N}^{N}\right)$. We now derive an a-priori estimate which allows us to infer global existence. Multiplying each equation of (2.1) by $-\lambda_{j} a_{j}(t)$ and summing (that is, using $u_{x x}^{N}$ as a test function), we get

$$
\frac{\mathrm{d}}{\mathrm{d} t} \frac{1}{2} \int_{\Omega}\left(u_{x}^{N}\right)^{2}+\varepsilon \int_{\Omega} q_{1}\left(u_{x x x}^{N}\right)^{2}=\int_{\Omega} q_{1} f_{1 u} u_{x}^{N} u_{x x x}^{N}+\int_{\Omega} q_{1} f_{1 v} v_{x}^{N} u_{x x x}^{N}
$$


using $v_{x x}^{N}$ as a test function in (2.2) we obtain

$$
\frac{\mathrm{d}}{\mathrm{d} t} \frac{1}{2} \int_{\Omega}\left(v_{x}^{N}\right)^{2}+\varepsilon \int_{\Omega} q_{2}\left(v_{x x}^{N}\right)^{2}=\int_{\Omega} q_{2} f_{2} v_{x x}^{N} .
$$

Summing the two identities, using (H1)-(H2), and applying the Cauchy-Schwarz and Young's inequalities

$$
\frac{\mathrm{d}}{\mathrm{d} t}\left[\int_{\Omega}\left(u_{x}^{N}\right)^{2}+\int_{\Omega}\left(v_{x}^{N}\right)^{2}\right]+\int_{\Omega}\left(u_{x x x}^{N}\right)^{2}+\int_{\Omega}\left(v_{x x}^{N}\right)^{2} \leqslant c_{1}+c_{2}\left[\int_{\Omega}\left(u_{x}^{N}\right)^{2}+\int_{\Omega}\left(v_{x}^{N}\right)^{2}\right],
$$

from which we obtain by a Gronwall argument

$$
\int_{\Omega}\left(u_{x}^{N}\right)^{2}+\int_{\Omega}\left(v_{x}^{N}\right)^{2}+\iint_{\Omega_{T}}\left(u_{x x x}^{N}\right)^{2}+\iint_{\Omega_{T}}\left(v_{x x}^{N}\right)^{2} \leqslant c_{3}(T) .
$$

Since $\psi_{1}^{\prime} \equiv 0$, it follows from (2.1) that $\int_{\Omega} u_{t}^{N}=0$; hence, by Poincaré inequality

$$
\left\|u^{N}\right\|_{L^{\infty}\left(0, T ; H^{1}(\Omega)\right)} \leqslant c_{4}(T)
$$

The relation

$$
\iint_{\Omega_{T}}\left(v_{t}^{N}\right)^{2}=-\iint_{\Omega_{T}} v_{t}^{N} q_{2}\left(f_{2}-\varepsilon v_{x x}^{N}\right) \leqslant\left(\iint_{\Omega_{T}}\left(v_{t}^{N}\right)^{2}\right)^{1 / 2}\left(\iint_{\Omega_{T}} q_{2}^{2}\left(f_{2}-\varepsilon v_{x x}^{N}\right)^{2}\right)^{1 / 2}
$$

coupled with (2.4) gives

$$
\left\|v_{t}^{N}\right\|_{L^{2}\left(\Omega_{T}\right)} \leqslant c_{5}(T)
$$

which in turn implies a bound on $b_{1}^{N}(t)$. Therefore, using the Poincaré inequality and (2.4) we have

$$
\begin{gathered}
\left\|v^{N}\right\|_{L^{\infty}\left(0, T ; H^{1}(\Omega)\right)} \leqslant c_{6}(T), \\
\left\|v^{N}\right\|_{L^{2}\left(0, T ; H^{2}(\Omega)\right)} \leqslant c_{6}(T) .
\end{gathered}
$$

From (2.5) and (2.7), it follows that $\left(a_{1}^{N}, \ldots, a_{N}^{N}, b_{1}^{N}, \ldots, b_{N}^{N}\right)$ are uniformly bounded, and therefore there exists a solution of $(2.1)-(2.3)$ in $(0, T)$.

In order to derive an estimate for $u_{t}^{N}$, we introduce the projection $\Pi_{N}$ of $L^{2}(\Omega)$ onto $\operatorname{span}\left\{\psi_{1}, \ldots, \psi_{N}\right\}$; for any $\psi \in L^{2}\left(0, T ; H^{1}(\Omega)\right)$ we obtain

$$
\left|\iint_{\Omega_{T}} u_{t}^{N} \psi\right|=\left|\iint_{\Omega_{T}} q_{1}\left(f_{1}-\varepsilon u_{x x}^{N}\right)_{x}\left(\Pi_{N} \psi\right)_{x}\right| \leqslant c_{7}(T)\|\psi\|_{L^{2}\left(0, T ; H^{1}(\Omega)\right)}
$$

(where (2.4) has been used); it follows that

$$
\left\|u_{t}^{N}\right\|_{L^{2}\left(0, T ;\left(H^{1}(\Omega)\right)^{\prime}\right)} \leqslant c_{7}(T) .
$$


Finally, since

$$
\int_{\Omega} u_{x x}^{N}=0
$$

the estimate (2.4), Poincaré's inequality and (2.5) yield

$$
\left\|u^{N}\right\|_{L^{2}\left(0, T ; H^{3}(\Omega)\right)} \leqslant c_{8}(T) .
$$

From the estimates (2.5)-(2.9) and (2.11), by well-known compactness results (see also [29]) it is possible to select a subsequence (still denoted by $\left.\left(u^{N}, v^{N}\right)\right)$ such that

$$
\begin{array}{ccccc}
u^{N}, v^{N} & -* & u, v & \text { in } & L^{\infty}\left(0, T ; H^{1}(\Omega)\right), \\
u^{N} & \longrightarrow & u & \text { in } & L^{2}\left(0, T ; H^{3}(\Omega)\right), \\
v^{N} & \longrightarrow & v & \text { in } & L^{2}\left(0, T ; H^{2}(\Omega)\right), \\
u_{t}^{N} & \longrightarrow & u_{t} & \text { in } & L^{2}\left(0, T ;\left(H^{1}(\Omega)\right)^{\prime}\right), \\
v_{t}^{N} & \longrightarrow & v_{t} & \text { in } & L^{2}\left(\Omega_{T}\right), \\
u^{N}, v^{N} & \longrightarrow & u, v & \text { in } & C\left([0, T] ; H^{\lambda}(\Omega)\right), \lambda<1, \lambda \\
u^{N} & \longrightarrow & u & \text { in } & L^{2}\left(0, T ; H^{\lambda}(\Omega)\right), \lambda<3, \\
v^{N} & \longrightarrow & v & \text { in } & L^{2}\left(0, T ; H^{\lambda}(\Omega)\right), \lambda<2 .
\end{array}
$$

In particular, the strong convergence in $C\left([0, T] ; L^{2}(\Omega)\right)$ implies (iv), and the last two convergence statements imply $(\mathrm{v})$ in view of the continuous linear mapping

$$
L^{2}\left(0, T ; H^{1-\lambda}(\Omega)\right) \rightarrow L^{2}\left(0, T ; H^{\frac{1}{2}-\lambda}(\partial \Omega)\right), \quad \lambda<\frac{1}{2}
$$

applied to $u_{x}^{N}, v_{x}^{N}$ with $\lambda>0$.

REMARK 2.2 Note that, using the same arguments as above with some minor modifications, Theorem 2.1 can be proved in any space dimension.

\section{Proof of Theorem 1}

\subsection{Approximating systems}

We shall approximate Problem $(\mathbf{P})$ by regularized systems; i.e. systems with positive mobility and smooth bulk energy. For $\delta>0$ we introduce positive mobilities $Q_{\delta}(u, v)$ by defining (see Fig. 1)

$$
\begin{aligned}
& Q_{2 \delta}(v)= \begin{cases}\left(\frac{1}{2}+\delta\right)^{2}-v^{2} & \text { if } \quad v \in\left[0, \frac{1}{2}+\frac{\delta}{2}\right], \\
\frac{\delta^{2}}{4}\left[1+(1+\delta)\left(v-\frac{1}{2}\right)^{-1}\right] & \text { if } \quad v>\frac{1}{2}+\frac{\delta}{2}, \\
Q_{2 \delta}(-v) & \text { if } \quad v<0,\end{cases} \\
& Q_{1 \delta}(u)=Q_{2 \delta}\left(u-\frac{1}{2}\right),
\end{aligned}
$$




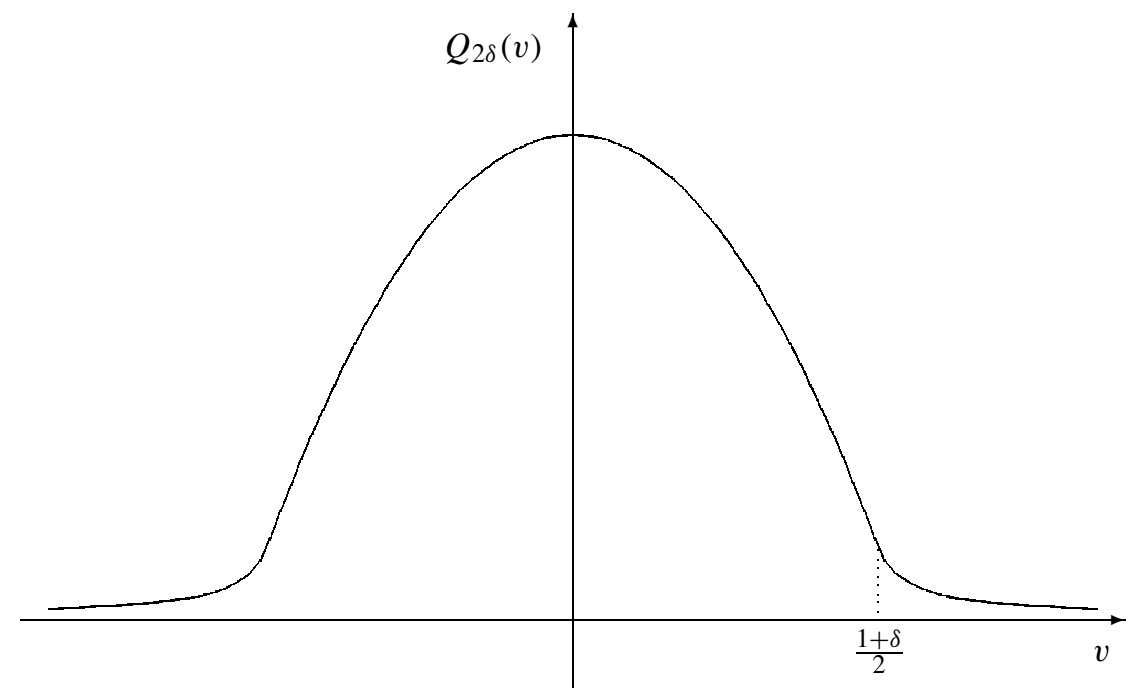

FIG. 1. The function $Q_{2 \delta}(v)$.

and setting

$$
Q_{\delta}(u, v)=Q_{1 \delta}(u) Q_{2 \delta}(v) .
$$

With this choice we have $Q_{i \delta}^{\prime \prime} \in L^{\infty}(\mathbb{R})$ and

$$
\begin{gathered}
\frac{\delta^{2}}{4}<Q_{i \delta} \leqslant\left(\frac{1}{2}+\delta\right)^{2} \\
\left|Q_{i \delta}^{\prime}\right| \leqslant 1+\delta \\
Q_{i \delta} \longrightarrow Q_{i} \quad \text { uniformly in } C^{1}\left(\operatorname{supp}\left[Q_{i}\right]_{+}\right) .
\end{gathered}
$$

For $\sigma \in(0,1 / e)$ we choose $F_{\sigma}^{\prime}(s)$ such that (see Fig. 2)

$$
F_{\sigma}^{\prime}(s)=\left\{\begin{array}{lll}
{\left[1-\frac{s-\sigma}{\sigma}\right]^{-1}+\ln \sigma,} & \text { if } \quad s<\sigma, \\
\ln s+1, & \text { if } \quad \sigma \leqslant s \leqslant 1-\sigma, \\
f_{\sigma}(s), & \text { if } \quad 1-\sigma<s<2, \\
1, & \text { if } \quad s \geqslant 2,
\end{array}\right.
$$

where $f_{\sigma} \in C^{1}([1-\sigma, 2])$ has the following properties:

$$
\begin{gathered}
f_{\sigma} \leqslant F^{\prime}, \quad f_{\sigma}^{\prime} \geqslant 0, \\
f_{\sigma}(1-\sigma)=F^{\prime}(1-\sigma), \quad f_{\sigma}(2)=1, \\
f_{\sigma}^{\prime}(1-\sigma)=F^{\prime \prime}(1-\sigma), \quad f_{\sigma}^{\prime}(2)=0 .
\end{gathered}
$$




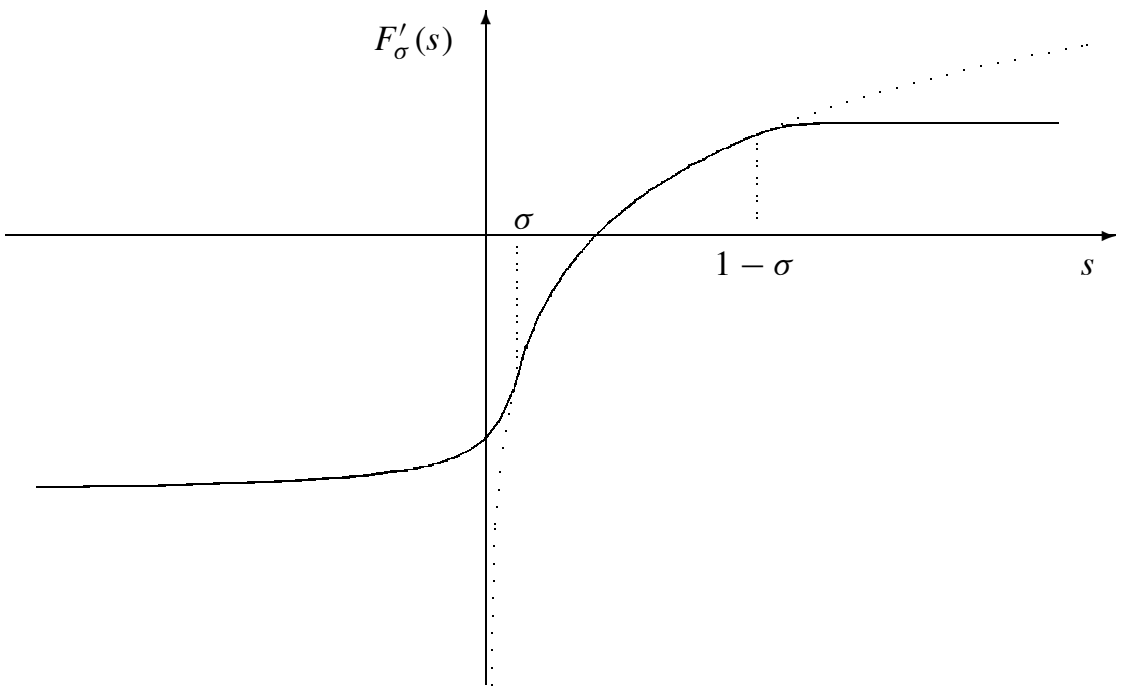

FIG. 2. The function $F_{\sigma}^{\prime}(s)$ (the dotted curve is $F^{\prime}(s)$ ).

Defining

$$
F_{\sigma}(s)=-\frac{1}{e}+\int_{\frac{1}{e}}^{s} F_{\sigma}^{\prime}(\xi) \mathrm{d} \xi
$$

we have

$$
\begin{aligned}
F_{\sigma} \in C^{2}(\mathbb{R}), & F_{\sigma}(s) \leqslant F(s) \text { if } 0<s, \\
F_{\sigma}^{\prime \prime} \geqslant 0, & F_{\sigma}(s)=F(s) \text { if } \sigma \leqslant s \leqslant 1-\sigma .
\end{aligned}
$$

Note that $F_{\sigma}$ is defined for all real values of $s$, whereas $F$ is defined for $s>0$ only. We also introduce $U, V$ such that

$$
\begin{array}{cl}
\|U\|_{C^{2}(\mathbb{R})} \leqslant U_{0}, & \|V\|_{C^{2}(\mathbb{R})} \leqslant V_{0}, \\
U(u)=\alpha u(1-u) \text { if } 0 \leqslant u \leqslant 1, & V(v)=-\beta v^{2} \text { if }-\frac{1}{2} \leqslant v \leqslant \frac{1}{2},
\end{array}
$$

and we define the approximating homogeneous free energies as follows:

$$
\mathcal{F}_{\sigma}(u, v)=F_{\sigma}(u+v)+F_{\sigma}(u-v)+F_{\sigma}(1-(u+v))+F_{\sigma}(1-(u-v))+U(u)+V(v) .
$$

We observe that

$$
-U_{0}-V_{0}-\frac{4}{e} \leqslant \mathcal{F}_{\sigma}(u, v) \text { in } \mathbb{R}^{2}, \quad \text { and } \mathcal{F}_{\sigma}(u, v)<U(u)+V(v) \text { in } \bar{B} .
$$

Applying Theorem 2.1, for each $\delta, \sigma>0$ there exists a solution $\left(u_{\delta \sigma}, v_{\delta \sigma}\right)$ of 


$$
\left(\mathbf{P}_{\delta \sigma}\right) \begin{cases}u_{t}=\left[Q_{\delta}(u, v)\left(\mathcal{F}_{\sigma u}(u, v)-\varepsilon u_{x x}\right)_{x}\right]_{x} & (x, t) \in \Omega_{T} \\ v_{t}=-Q_{\delta}(u, v)\left(\mathcal{F}_{\sigma v}(u, v)-\varepsilon v_{x x}\right) & (x, t) \in \Omega_{T} \\ u_{x}=v_{x}=Q_{\delta}(u, v)\left(\mathcal{F}_{\sigma u}(u, v)-u_{x x}\right)_{x}=0 & (x, t) \in S_{T} \\ u(x, 0)=u_{0}(x), \quad v(x, 0)=v_{0}(x) & x \in \Omega .\end{cases}
$$

We shall hereafter denote by $\left(\mathbf{P}_{\delta}\right)$, Problem $\left(\mathbf{P}_{\delta \sigma}\right)$ where $\mathcal{F}_{\sigma}$ is replaced by $\mathcal{F}$, and we shall denote by $\delta_{0}, \sigma_{0}$ generic positive constants.

First of all we observe that

$$
\overline{u_{\delta \sigma}(t)}=\overline{u_{0}} \in(0,1) .
$$

LEMmA 3.1 There exists a constant $C_{1}$ independent of $\delta, \sigma$ such that the following estimates hold for all $\delta<\delta_{0}, \sigma<\sigma_{0}$ :

(i) $\left\|u_{\delta \sigma}\right\|_{L^{\infty}\left(0, T ; H^{1}(\Omega)\right)} \leqslant C_{1}$,

(ii) $\left\|v_{\delta \sigma}\right\|_{L^{\infty}\left(0, T ; H^{1}(\Omega)\right)} \leqslant C_{1}$,

(iii) $\left\|Q_{\delta}^{1 / 2}\left(\mathcal{F}_{\sigma u}-\varepsilon u_{\delta \sigma x x}\right)_{x}\right\|_{L^{2}\left(\Omega_{T}\right)} \leqslant C_{1}$,

(iv) $\left\|Q_{\delta}^{1 / 2}\left(\mathcal{F}_{\sigma v}-\varepsilon v_{\delta \sigma x x}\right)\right\|_{L^{2}\left(\Omega_{T}\right)} \leqslant C_{1}$,

(v) $\left\|u_{\delta \sigma t}\right\|_{L^{2}\left(0, T ;\left(H^{1}(\Omega)\right)^{\prime}\right)} \leqslant C_{1}$,

(vi) $\left\|Q_{\delta}^{-1 / 2} v_{\delta \sigma t}\right\|_{L^{2}\left(\Omega_{T}\right)} \leqslant C_{1}$,

(vii) $\left\|\mathcal{F}_{\sigma}\left(u_{\delta \sigma}, v_{\delta \sigma}\right)\right\|_{L^{\infty}\left(0, T ; L^{1}(\Omega)\right)} \leqslant C_{1}$.

Proof. Choosing $\phi=\left(\mathcal{F}_{\sigma u}-\varepsilon u_{\delta \sigma x x}\right)$ and $\psi=\left(\mathcal{F}_{\sigma v}-\varepsilon v_{\delta \sigma x x}\right)$ as test functions in the equations for $u_{\delta \sigma}$ and $v_{\delta \sigma}$ respectively, we obtain

$$
\begin{gathered}
\frac{\varepsilon}{2} \int_{\Omega}\left[u_{\delta \sigma x}(t)\right]^{2}+\frac{\varepsilon}{2} \int_{\Omega}\left[v_{\delta \sigma x}(t)\right]^{2}+\int_{\Omega} \mathcal{F}_{\sigma}\left(u_{\delta \sigma}(t), v_{\delta \sigma}(t)\right) \\
+\iint_{\Omega_{t}} Q_{\delta}\left[\left(\mathcal{F}_{\sigma u}-\varepsilon u_{\delta \sigma x x}\right)_{x}\right]^{2}+\iint_{\Omega_{t}} Q_{\delta}\left(\mathcal{F}_{\sigma v}-\varepsilon v_{\delta \sigma x x}\right)^{2} \\
=\frac{\varepsilon}{2} \int_{\Omega}\left(u_{0 x}\right)^{2}+\frac{\varepsilon}{2} \int_{\Omega}\left(v_{0 x}\right)^{2}+\int_{\Omega} \mathcal{F}_{\sigma}\left(u_{0}, v_{0}\right)
\end{gathered}
$$

for almost every $t \in(0, T]$ (see [19] for a detailed proof). Using (1.4) and (3.1) we obtain

$$
\begin{aligned}
& \frac{\varepsilon}{2} \int_{\Omega}\left[u_{\delta \sigma x}(t)\right]^{2}+\frac{\varepsilon}{2} \int_{\Omega}\left[v_{\delta \sigma x}(t)\right]^{2}+\int_{\Omega} \mathcal{F}_{\sigma}\left(u_{\delta \sigma}(t), v_{\delta \sigma}(t)\right) \\
& \quad+\iint_{\Omega_{t}} Q_{\delta}\left[\left(\mathcal{F}_{\sigma u}-\varepsilon u_{\delta \sigma x x}\right)_{x}\right]^{2}+\iint_{\Omega_{t}} Q_{\delta}\left(\mathcal{F}_{\sigma v}-\varepsilon v_{\delta \sigma x x}\right)^{2} \leqslant c_{9}
\end{aligned}
$$

which implies (iii), (iv) and (vii) since $-U_{0}-V_{0}-\frac{4}{e} \leqslant \mathcal{F}_{\sigma}$. Using the Poincaré inequality and (3.2), (i) is also verified. To prove (vi), we choose $\psi=v_{\delta \sigma t} / Q_{\delta}\left(u_{\delta \sigma}, v_{\delta \sigma}\right)$ as a test function in the 
second equation of $\left(\mathbf{P}_{\delta \sigma}\right)$, which yields

$$
\iint_{\Omega_{T}} \frac{\left(v_{\delta \sigma t}\right)^{2}}{Q_{\delta}\left(u_{\delta \sigma}, v_{\delta \sigma}\right)}=-\iint_{\Omega_{T}}\left(\mathcal{F}_{\sigma v}-\varepsilon v_{\delta \sigma x x}\right) v_{\delta \sigma t} \leqslant\left(\iint_{\Omega_{T}} Q_{\delta}\left(\mathcal{F}_{\sigma v}-\varepsilon v_{\delta \sigma x x}\right)^{2}\right)^{1 / 2}\left(\iint_{\Omega_{T}} \frac{\left(v_{\delta \sigma t}\right)^{2}}{Q_{\delta}}\right)^{1 / 2} .
$$

Since $Q_{\delta} \leqslant 1$ for $\delta$ small enough, in particular it holds that

$$
\int_{\Omega} v_{\delta \sigma}^{2}(t) \leqslant 2 \int v_{\Omega} v_{0}^{2}+2 t \iint_{\Omega_{t}}\left(v_{\delta \sigma t}\right)^{2} \leqslant c_{10}
$$

which together with (3.3) implies (ii). Finally, (v) follows since

$$
\left|\iint_{\Omega_{T}} u_{\delta \sigma t} \phi\right| \leqslant\left(\iint_{\Omega_{T}} Q_{\delta}\left[\left(\mathcal{F}_{\sigma u}-\varepsilon u_{\delta \sigma x x}\right)_{x}\right]^{2}\right)^{1 / 2}\left(\iint_{\Omega_{T}}\left(\phi_{x}\right)^{2}\right)^{1 / 2}
$$

for all $\phi \in L^{2}\left(0, T ; H^{1}(\Omega)\right)$.

Arguing in a standard fashion (see [5] for a proof) we have

COROLlary 3.2 There exists a constant $C_{2}$ independent of $0<\sigma<\sigma_{0}, 0<\delta<\delta_{0}$ such that

$$
\begin{gathered}
\left\|u_{\delta \sigma}\right\|_{C^{0, \frac{1}{2}, \frac{1}{8}}\left(\bar{\Omega}_{T}\right)} \leqslant C_{2}, \\
\left\|v_{\delta \sigma}\right\|_{C^{0, \frac{1}{2}, \frac{1}{4}}\left(\bar{\Omega}_{T}\right)} \leqslant C_{2} .
\end{gathered}
$$

\subsection{Systems with positive mobility}

Throughout this subsection we fix $\delta>0$, and assume the mobility to be given by the form $Q_{\delta}(u, v)$ introduced in Subsection 3.1. However, we remark that the results in this subsection are independent of the particular form of the mobility, and hold for any $\tilde{Q}(u, v) \in C\left(\mathbb{R}^{2} ; \mathbb{R}\right)$ with $0<Q_{\min } \leqslant \tilde{Q} \leqslant$ $Q_{\max }$.

By Corollary 3.2 we can extract a subsequence (still denoted by $\left.\left(u_{\delta \sigma}, v_{\delta \sigma}\right)\right)$ such that

$$
\begin{aligned}
& \left(u_{\delta \sigma}, v_{\delta \sigma}\right) \rightarrow\left(u_{\delta}, v_{\delta}\right) \text { uniformly in } \bar{\Omega}_{T} \text { as } \sigma \rightarrow 0 \\
& u_{\delta} \in C^{0, \frac{1}{2}, \frac{1}{8}}\left(\bar{\Omega}_{T}\right), \quad v_{\delta} \in C^{0, \frac{1}{2}, \frac{1}{4}}\left(\bar{\Omega}_{T}\right) .
\end{aligned}
$$

We now demonstrate that the limit $\left(u_{\delta}, v_{\delta}\right)$ lies within the square $B$.

LEMMA 3.3 For all $0<\delta<\delta_{0}$,

$$
\left|\Omega_{T} \backslash \mathcal{B}\left(u_{\delta}, v_{\delta}\right)\right|=0 .
$$

Proof. Let $N$ denote minus the inverse of the Laplacian with zero Neumann boundary conditions: given $f \in\left(H^{1}(\Omega)\right)_{\text {null }}^{\prime}:=\left\{f \in\left(H^{1}(\Omega)\right)^{\prime}:\langle f, 1\rangle=0\right\}$, we define $N f \in H^{1}(\Omega)$ as the unique 
solution of

$$
\begin{aligned}
& \int_{\Omega}(N f)^{\prime} \psi^{\prime}=\langle f, \psi\rangle \quad \forall \psi \in H^{1}(\Omega) \\
& \int_{\Omega} N f=0 .
\end{aligned}
$$

By (3.2) and Lemma 3.1 (i), $N\left(u_{\delta \sigma}(t)-\overline{u_{\delta \sigma}}\right)$ is well defined. Choosing $\phi=Q_{\delta}^{-1} N\left(u_{\delta \sigma}-\overline{u_{\delta \sigma}}\right)$ as a test function in the equation for $u_{\delta \sigma}$, we have

$$
\begin{aligned}
\int_{0}^{T}\left\langle u_{\delta \sigma t},\right. & \left.Q_{\delta}^{-1} N\left(u_{\delta \sigma}-\overline{u_{\delta \sigma}}\right)\right\rangle \mathrm{d} t= \\
& =-\iint_{\Omega_{T}}\left(\mathcal{F}_{\sigma u}-\varepsilon u_{\delta \sigma x x}\right)_{x}\left(N\left(u_{\delta \sigma}-\overline{u_{\delta \sigma}}\right)\right)_{x} \\
& +\iint_{\Omega_{T}} Q_{\delta}^{-1}\left(\mathcal{F}_{\sigma u}-\varepsilon u_{\delta \sigma x x}\right)_{x} Q_{\delta x} N\left(u_{\delta \sigma}-\overline{u_{\delta \sigma}}\right) \\
& =-\iint_{\Omega_{T}}\left(u_{\delta \sigma}-\overline{u_{\delta \sigma}}\right) \mathcal{F}_{\sigma u}-\varepsilon \iint_{\Omega_{T}}\left(u_{\delta \sigma x}\right)^{2} \\
& +\iint_{\Omega_{T}} Q_{\delta}^{-1}\left(\mathcal{F}_{\sigma u}-\varepsilon u_{\delta \sigma x x}\right)_{x} Q_{\delta x} N\left(u_{\delta \sigma}-\overline{u_{\delta \sigma}}\right) .
\end{aligned}
$$

Choosing $\psi=Q_{\delta}^{-1} v_{\delta \sigma}$ as a test function in the equation for $v_{\delta \sigma}$,

$$
\iint_{\Omega_{T}} Q_{\delta}^{-1} v_{\delta \sigma} v_{\delta \sigma t}=-\iint_{\Omega_{T}} v_{\delta \sigma} \mathcal{F}_{\sigma v}-\varepsilon \iint_{\Omega_{T}}\left(v_{\delta \sigma x}\right)^{2} .
$$

Summing the two above identities, we obtain

$$
\begin{aligned}
& \iint_{\Omega_{T}}\left(u_{\delta \sigma}-\overline{u_{\delta \sigma}}\right) \mathcal{F}_{\sigma u}+\iint_{\Omega_{T}} v_{\delta \sigma} \mathcal{F}_{\sigma v} \\
& =-\int_{0}^{T}\left\langle u_{\delta \sigma t}, Q_{\delta}^{-1} N\left(u_{\delta \sigma}-\overline{u_{\delta \sigma}}\right)\right\rangle \mathrm{d} t-\varepsilon \iint_{\Omega_{T}}\left[\left(u_{\delta \sigma x}\right)^{2}+\left(v_{\delta \sigma x}\right)^{2}\right] \\
& \quad+\iint_{\Omega_{T}} Q_{\delta}^{-1}\left(\mathcal{F}_{\sigma u}-\varepsilon u_{\delta \sigma x x}\right)_{x} Q_{\delta x} N\left(u_{\delta \sigma}-\overline{u_{\delta \sigma}}\right)-\iint_{\Omega_{T}} Q_{\delta}^{-1} v_{\delta \sigma} v_{\delta \sigma t} .
\end{aligned}
$$

The estimates in Lemma 3.1, the lower bound for $Q_{\delta}$, the fact that $\left\|Q_{\delta}\right\|_{C^{1}} \leqslant 2$ for $\delta$ sufficiently small, and the definition of $N$ imply

$$
\iint_{\Omega_{T}}\left[\left(u_{\delta \sigma}-\overline{u_{\delta \sigma}}\right) \mathcal{F}_{\sigma u}+v_{\delta \sigma} \mathcal{F}_{\sigma v}\right] \leqslant c_{11}
$$


(where $c_{11}=c_{11}(\delta)$ ). We now exploit the sign property of the integrand at the left hand side. To this purpose, we observe that the following identity holds for any $c \in \mathbb{R}$ :

$$
\begin{aligned}
(u-c) \mathcal{F}_{\sigma u}(u, v)+v \mathcal{F}_{\sigma v} & (u, v)=\left\{(u+v)\left[F_{\sigma}^{\prime}(u+v)-1\right]+(u-v)\left[F_{\sigma}^{\prime}(u-v)-1\right]\right. \\
& +(1-(u+v))\left[F_{\sigma}^{\prime}(1-(u+v))-1\right] \\
& \left.+(1-(u-v))\left[F_{\sigma}^{\prime}(1-(u-v))-1\right]\right\} \\
& -c F_{\sigma}^{\prime}(u+v)-c F_{\sigma}^{\prime}(u-v)-(1-c) F_{\sigma}^{\prime}(1-(u+v)) \\
& -(1-c) F_{\sigma}^{\prime}(1-(u-v))+(u-c) U^{\prime}(u)+v V^{\prime}(v)+2 .
\end{aligned}
$$

The terms inside the braces are bounded from below since

$$
\begin{aligned}
0 & \leqslant s\left[F_{\sigma}^{\prime}(s)-1\right], & & s \leqslant 0, \\
-\frac{1}{e}-\frac{1}{2} & \leqslant \sigma[\ln \sigma-1] \leqslant s\left[F_{\sigma}^{\prime}(s)-1\right] \leqslant 0, & & 0<s \leqslant \sigma, \\
-\frac{1}{e} & \leqslant s \ln s=s\left[F_{\sigma}^{\prime}(s)-1\right] \leqslant 0, & & \sigma \leqslant s \leqslant 1-\sigma, \\
-2 & \leqslant s\left[F_{\sigma}^{\prime}(s)-1\right] \leqslant 0, & & 1-\sigma \leqslant s \leqslant 2, \\
0 & =s\left[F_{\sigma}^{\prime}(s)-1\right], & & s \geqslant 2
\end{aligned}
$$

for $\sigma \in(0,1 / 2)$. Since $U^{\prime}, V^{\prime}$ are uniformly bounded, setting $c=\overline{u_{\delta \sigma}} \equiv \overline{u_{0}} \in(0,1)$ in (3.7) and noting that $F_{\sigma}^{\prime} \leqslant 1$, it follows from (3.6) that

$$
\begin{aligned}
& -\iint_{\Omega_{T}}\left[F_{\sigma}^{\prime}\left(u_{\delta \sigma}+v_{\delta \sigma}\right)+F_{\sigma}^{\prime}\left(u_{\delta \sigma}-v_{\delta \sigma}\right)\right. \\
& \left.\quad+F_{\sigma}^{\prime}\left(1-\left(u_{\delta \sigma}+v_{\delta \sigma}\right)\right)+F_{\sigma}^{\prime}\left(1-\left(u_{\delta \sigma}-v_{\delta \sigma}\right)\right)\right] \leqslant c_{12} .
\end{aligned}
$$

To complete the proof, suppose by contradiction $\left|\Omega_{T} \backslash \mathcal{B}\left(u_{\delta}, v_{\delta}\right)\right|>0$. Since the argument can be repeated for each side of the box $B$, we can assume without loss of generality that the set

$$
A=\left\{(x, t) \in \Omega_{T}: u_{\delta}+v_{\delta} \leqslant 0\right\}
$$

has positive measure. Since $F_{\sigma}^{\prime} \leqslant 1$, the estimate (3.8) gives

$$
-\iint_{A} F_{\sigma}^{\prime}\left(u_{\delta \sigma}+v_{\delta \sigma}\right) \leqslant c_{13}
$$

Note, however, that the uniform convergence of $u_{\delta \sigma}$ and $v_{\delta \sigma}$ implies that

$$
\forall \lambda>0 \exists \sigma_{\lambda}: u_{\delta \sigma}+v_{\delta \sigma} \leqslant \lambda \quad \forall x \in A, \sigma<\sigma_{\lambda} ;
$$

therefore, due to the convexity of $F_{\sigma}, F_{\sigma}^{\prime}\left(u_{\delta \sigma}+v_{\delta \sigma}\right) \leqslant F_{\sigma}^{\prime}(\lambda)$. Hence

$$
c_{13} \geqslant-\lim _{\sigma \rightarrow 0} \iint_{A} F_{\sigma}^{\prime}(\lambda)=-|A|(\ln \lambda+1),
$$

which leads to a contradiction for $\lambda$ sufficiently small.

In the next Lemma we derive additional estimates which will allow us to pass to the limit as $\sigma \rightarrow 0$ in Problem $\left(\mathbf{P}_{\delta \sigma}\right)$. To simplify the notation we define the function

$$
\varphi_{\sigma}(s)=F_{\sigma}^{\prime \prime}(s)+F_{\sigma}^{\prime \prime}(1-s),
$$

which is positive in view of the definition of $F_{\sigma}^{\prime}$. 
Lemma 3.4 Let $\delta>0$ be fixed. Then there exists a constant $C_{3}$ which is independent of $\sigma$ such that

(i) $\left\|\mathcal{F}_{\sigma v}\left(u_{\delta \sigma}, v_{\delta \sigma}\right)\right\|_{L^{2}\left(\Omega_{T}\right)} \leqslant C_{3}$,

(ii) $\left\|u_{\delta \sigma x x}\right\|_{L^{2}\left(\Omega_{T}\right)} \leqslant C_{3}$,

(iii) $\left\|v_{\delta \sigma x x}\right\|_{L^{2}\left(\Omega_{T}\right)} \leqslant C_{3}$,

(iv) $\iint_{\Omega_{T}} \varphi_{\sigma}\left(u_{\delta \sigma}+v_{\delta \sigma}\right)\left[\left(u_{\delta \sigma}+v_{\delta \sigma}\right)_{x}\right]^{2}+\iint_{\Omega_{T}} \varphi_{\sigma}\left(u_{\delta \sigma}-v_{\delta \sigma}\right)\left[\left(u_{\delta \sigma}-v_{\delta \sigma}\right)_{x}\right]^{2} \leqslant C_{3}$.

Proof. From Lemma 3.1 (iv) we have

$$
\iint_{\Omega_{T}}\left(\mathcal{F}_{\sigma v}\right)^{2}+2 \varepsilon \iint_{\Omega_{T}}\left(\mathcal{F}_{\sigma v}\right)_{x} v_{\delta \sigma x}+\varepsilon^{2} \iint_{\Omega_{T}}\left(v_{\delta \sigma x x}\right)^{2} \leqslant c_{14}
$$

Defining $H_{\delta \sigma}=\mathcal{F}_{\sigma u}-\varepsilon u_{\delta \sigma x x}$, since $\left.u_{\delta \sigma x}\right|_{S_{T}}=0$

$$
\int_{\Omega} H_{\delta \sigma}(t)=\int_{\Omega} \mathcal{F}_{\sigma u}\left(u_{\delta \sigma}(t), v_{\delta \sigma}(t)\right)
$$

and from Lemma 3.1 (iii),

$$
\iint_{\Omega_{T}}\left(H_{\delta \sigma x}\right)^{2} \leqslant c_{15}
$$

Hence we can write

$$
\begin{aligned}
\iint_{\Omega_{T}}\left(\mathcal{F}_{\sigma u}\right)^{2} & +2 \varepsilon \iint_{\Omega_{T}}\left(\mathcal{F}_{\sigma u}\right)_{x} u_{\delta \sigma x}+\varepsilon^{2} \iint_{\Omega_{T}}\left(u_{\delta \sigma x x}\right)^{2}=\iint_{\Omega_{T}} H_{\delta \sigma}^{2} \\
= & \iint_{\Omega_{T}}\left(H_{\delta \sigma}-\overline{H_{\delta \sigma}}\right)^{2}+\iint_{\Omega_{T}} \bar{H}_{\delta \sigma}^{2} \leqslant c_{P} \iint_{\Omega_{T}}\left(H_{\delta \sigma x}\right)^{2}+\iint_{\Omega_{T}}\left(\mathcal{F}_{\sigma u}\right)^{2},
\end{aligned}
$$

where $c_{P}$ denotes the Poincaré coefficient. Summing this inequality with (3.9) we obtain

$$
\iint_{\Omega_{T}}\left(\left(\mathcal{F}_{\sigma v}\right)^{2}+2 \varepsilon \iint_{\Omega_{T}}\left[\left(\mathcal{F}_{\sigma u}\right)_{x} u_{\delta \sigma x}+\left(\mathcal{F}_{\sigma v}\right)_{x} v_{\delta \sigma x}\right]+\varepsilon^{2} \iint_{\Omega_{T}}\left[\left(u_{\delta \sigma x x}\right)^{2}+\left(v_{\delta \sigma x x}\right)^{2}\right] \leqslant c_{16} .\right.
$$

From this estimate, the Lemma follows recalling that $\varphi_{\sigma}>0$ and observing that

$$
\begin{aligned}
\left(\mathcal{F}_{\sigma u}\right)_{x} u_{\delta \sigma x}+\left(\mathcal{F}_{\sigma v}\right)_{x} v_{\delta \sigma x} & =\varphi_{\sigma}\left(u_{\delta \sigma}+v_{\delta \sigma}\right)\left[\left(u_{\delta \sigma}+v_{\delta \sigma}\right)_{x}\right]^{2} \\
& +\varphi_{\sigma}\left(u_{\delta \sigma}-v_{\delta \sigma}\right)\left[\left(u_{\delta \sigma}-v_{\delta \sigma}\right)_{x}\right]^{2} \\
& +U^{\prime \prime}\left(u_{\delta \sigma}\right)\left(u_{\delta \sigma x}\right)^{2}+V^{\prime \prime}\left(v_{\delta \sigma}\right)\left(v_{\delta \sigma x}\right)^{2}
\end{aligned}
$$

and using the definition of $U, V$ and Lemma 3.1 (i), (ii) to bound the last two terms.

We can now state the following result. 
PRoposition 3.5 For each $0<\delta<\delta_{0}$ there exists a triplet $\left(u_{\delta}, v_{\delta}, w_{\delta}\right)$ such that:

(i) $u_{\delta}, v_{\delta} \in L^{\infty}\left(0, T ; H^{1}(\Omega)\right)$ uniformly in $\delta$;

(ii) $u_{\delta} \in C^{0, \frac{1}{2}, \frac{1}{8}}\left(\bar{\Omega}_{T}\right)$ and $v_{\delta} \in C^{0, \frac{1}{2}, \frac{1}{4}}\left(\bar{\Omega}_{T}\right)$ uniformly in $\delta$;

(iii) $u_{\delta t} \in L^{2}\left(0, T ;\left(H^{1}(\Omega)\right)^{\prime}\right)$ and $Q_{\delta}^{1 / 2} v_{\delta t} \in L^{2}\left(\Omega_{T}\right)$ uniformly in $\delta$;

(iv) $u_{\delta x x}, v_{\delta x x} \in L^{2}\left(\Omega_{T}\right)$;

(v) $\left|\Omega_{T} \backslash \mathcal{B}\left(u_{\delta}, v_{\delta}\right)\right|=0$;

(vi) $\mathcal{F}_{v}\left(u_{\delta}, v_{\delta}\right) \in L^{2}\left(\Omega_{T}\right)$ and $\mathcal{F}_{u}\left(u_{\delta}, v_{\delta}\right) \in L_{\text {loc }}^{2}\left(\mathcal{D}\left(u_{\delta}\right)\right)$;

(vii) $w_{\delta} \in L^{2}\left(\Omega_{T}\right)$ uniformly in $\delta$;

(viii) $u_{\delta}(x, 0)=u_{0}(x), v_{\delta}(x, 0)=v_{0}(x)$;

(ix) $\left.u_{\delta x}\right|_{S_{T}}=\left.v_{\delta x}\right|_{S_{T}}=0$ in $L^{2}\left(S_{T}\right)$;

(x) $\left(u_{\delta}, v_{\delta}, w_{\delta}\right)$ solves $\left(\mathbf{P}_{\delta}\right)$ in the following sense:

$$
\begin{aligned}
& \int_{0}^{T}\left\langle u_{\delta t}, \phi\right\rangle \mathrm{d} t=-\iint_{\Omega_{T}} Q_{\delta}^{1 / 2}\left(u_{\delta}, v_{\delta}\right) w_{\delta} \phi_{x} \quad \forall \phi \in L^{2}\left(0, T ; H^{1}(\Omega)\right), \\
& \iint_{\Omega_{T}} v_{\delta t} \psi=-\iint_{\Omega_{T}} Q_{\delta}\left(u_{\delta}, v_{\delta}\right)\left(\mathcal{F}_{v}\left(u_{\delta}, v_{\delta}\right)-\varepsilon v_{\delta x x}\right) \psi \quad \forall \psi \in L^{2}\left(\Omega_{T}\right),
\end{aligned}
$$

where $w_{\delta}=Q_{\delta}^{1 / 2}\left(\mathcal{F}_{u}-\varepsilon u_{\delta x x}\right)_{x}$ in the sense that

$$
\iint_{\Omega_{T}} w_{\delta} \phi=-\iint_{\Omega_{T}}\left(\mathcal{F}_{u}\left(u_{\delta}, v_{\delta}\right)-\varepsilon u_{\delta x x}\right)\left(Q_{\delta}^{1 / 2}\left(u_{\delta}, v_{\delta}\right) \phi\right)_{x}
$$

for all $\phi \in \longleftarrow^{2}\left(0, T ; H_{0}^{1}(\Omega)\right)$ with compact support in $\mathcal{D}\left(u_{\delta}\right)$.

Proof. Let $\left\{\left(u_{\delta \sigma}, v_{\delta \sigma}\right)\right\}$ be the sequence given in (3.5). By Lemmas 3.1 and 3.4, it can be seen that

$$
\begin{aligned}
& u_{\delta \sigma}, v_{\delta \sigma} \quad \stackrel{*}{\rightarrow} u_{\delta}, v_{\delta} \quad \text { in } L^{\infty}\left(0, T ; H^{1}(\Omega)\right) \text {, } \\
& u_{\delta \sigma}, v_{\delta \sigma} \quad \longrightarrow \quad u_{\delta}, v_{\delta} \quad \text { in } \quad L^{2}\left(0, T ; H^{2}(\Omega)\right) \text {, } \\
& u_{\delta \sigma t} \longrightarrow u_{\delta t} \quad \text { in } L^{2}\left(0, T ;\left(H^{1}(\Omega)\right)^{\prime}\right) \text {, } \\
& Q_{\delta}^{-1 / 2}\left(u_{\delta \sigma}, v_{\delta \sigma}\right) v_{\delta \sigma t} \quad \longrightarrow \quad Q_{\delta}^{-1 / 2}\left(u_{\delta}, v_{\delta}\right) v_{\delta t} \quad \text { in } \quad L^{2}\left(\Omega_{T}\right) \text {, } \\
& Q_{\delta}^{1 / 2}\left(u_{\delta \sigma}, v_{\delta \sigma}\right)\left(\mathcal{F}_{\sigma u}-\varepsilon u_{\delta \sigma x x}\right)_{x} \longrightarrow w_{\delta} \quad \text { in } L^{2}\left(\Omega_{T}\right) \text {. }
\end{aligned}
$$

Recalling also Corollary 3.2 and Lemma 3.3, (i)-(v) and (vii) now follow. Lemma 3.4 (i) implies that

$$
\mathcal{F}_{\sigma v}\left(u_{\delta \sigma}, v_{\delta \sigma}\right) \longrightarrow g \text { in } L^{2}\left(\Omega_{T}\right) ;
$$

since $\mathcal{F}_{\sigma v}\left(u_{\delta \sigma}, v_{\delta \sigma}\right)$ converges pointwise in $\mathcal{B}\left(u_{\delta}, v_{\delta}\right)$ and $\left|\Omega_{T} \backslash \mathcal{B}\left(u_{\delta}, v_{\delta}\right)\right|=0$, it follows that $g=\mathcal{F}_{v}\left(u_{\delta}, v_{\delta}\right)$, which proves the first part of (vi). In order to prove the second part, we recall that

$$
\begin{aligned}
& \mathcal{F}_{\sigma u}(u, v)=F_{\sigma}^{\prime}(u+v)+F_{\sigma}^{\prime}(u-v)-F_{\sigma}^{\prime}(1-(u+v))-F_{\sigma}^{\prime}(1-(u-v))+U^{\prime}(u) \\
& \mathcal{F}_{\sigma v}(u, v)=F_{\sigma}^{\prime}(u+v)-F_{\sigma}^{\prime}(u-v)-F_{\sigma}^{\prime}(1-(u+v))+F_{\sigma}^{\prime}(1-(u-v))+V^{\prime}(v) .
\end{aligned}
$$


Lemma 3.4 (i) and the structure of $\mathcal{F}_{\sigma v}$ yield a uniform bound in $L^{2}\left(\mathcal{D}\left(u_{\delta}\right)\right)$ for each of its terms. Then this implies in turn a uniform bound in $L^{2}\left(\mathcal{D}\left(u_{\delta}\right)\right)$ for each of the terms in $\mathcal{F}_{\sigma u}$ : we prove this in detail for the first term. Let $K \subset \mathcal{D}\left(u_{\delta}\right)$ be compact; then

$$
K \subset\left\{\eta<u_{\delta}<1-\eta\right\}, \quad \frac{\eta}{2}<u_{\delta \sigma}<1-\frac{\eta}{2} \quad \text { in } K
$$

for a suitable $\eta>0$ and for $\sigma$ sufficiently small. Let us consider the sets

$$
K_{\sigma}^{\prime}=K \cap\left\{u_{\delta \sigma}+v_{\delta \sigma}<\eta / 2\right\}, \quad K_{\sigma}^{\prime \prime}=K \backslash K_{\sigma}^{\prime} ;
$$

in $K_{\sigma}^{\prime \prime}$ we have

$$
0 \geqslant F_{\sigma}^{\prime}\left(u_{\delta \sigma}+v_{\delta \sigma}\right)-1 \geqslant F_{\sigma}^{\prime}(\eta / 2)-1 \geqslant c_{17} .
$$

In order to estimate $F_{\sigma}^{\prime}\left(u_{\delta \sigma}+v_{\delta \sigma}\right)$ in $K_{\sigma}^{\prime}$, observe that the following relation holds:

$$
\begin{aligned}
0 \geqslant F_{\sigma}^{\prime}(u+v) & -1 \geqslant F_{\sigma}^{\prime}(u+v)+F_{\sigma}^{\prime}(1-(u-v))-2 \\
& =\mathcal{F}_{\sigma v}+F_{\sigma}^{\prime}(u-v)+F_{\sigma}^{\prime}(1-(u+v))-V^{\prime}(v)-2 .
\end{aligned}
$$

From its definition, in $K_{\sigma}^{\prime}$ we have $u_{\delta \sigma}-v_{\delta \sigma}>\eta / 2$ and $1-\left(u_{\delta \sigma}+v_{\delta \sigma}\right)>1-\eta / 2$; hence

$$
\begin{aligned}
0 \geqslant F_{\sigma}^{\prime}\left(u_{\delta \sigma}+v_{\delta \sigma}\right)-1 & \geqslant \mathcal{F}_{\sigma v}\left(u_{\delta \sigma}, v_{\delta \sigma}\right)+F_{\sigma}^{\prime}(\eta / 2)+F_{\sigma}^{\prime}(1-\eta / 2)-V^{\prime}\left(v_{\delta \sigma}\right)-2 \\
& \geqslant \mathcal{F}_{\sigma v}\left(u_{\delta \sigma}, v_{\delta \sigma}\right)+c_{18}
\end{aligned}
$$

which, together with (3.14), implies

$$
\iint_{K}\left[F_{\sigma}^{\prime}\left(u_{\delta \sigma}+v_{\delta \sigma}\right)-1\right]^{2} \leqslant c_{19}+2 \iint_{\Omega_{T}}\left(\mathcal{F}_{\sigma v}\left(u_{\delta \sigma}, v_{\delta \sigma}\right)\right)^{2} .
$$

Repeating the same argument for the other terms, we conclude that

$$
\iint_{K} \mathcal{F}_{\sigma u}^{2}\left(u_{\delta \sigma}, v_{\delta \sigma}\right) \leqslant c_{20}+8 \iint_{\Omega_{T}}\left(\mathcal{F}_{\sigma v}\left(u_{\delta \sigma}, v_{\delta \sigma}\right)\right)^{2}
$$

and therefore

$$
\mathcal{F}_{\sigma u}\left(u_{\delta \sigma}, v_{\delta \sigma}\right) \longrightarrow \mathcal{F}_{u}\left(u_{\delta}, v_{\delta}\right) \text { in } L_{\text {loc }}^{2}\left(\mathcal{D}\left(u_{\delta}\right)\right),
$$

which completes the proof of (vi).

The property (viii) is straightforward; by compactness we have that

$$
\begin{gathered}
u_{\delta \sigma} \longrightarrow u_{\delta} \text { in } L^{2}\left(0, T ; H^{2-\lambda}(\Omega)\right), \quad \lambda>0 \\
v_{\delta \sigma} \longrightarrow v_{\delta} \text { in } L^{2}\left(0, T ; H^{2-\lambda}(\Omega)\right), \quad \lambda>0
\end{gathered}
$$

which imply (ix).

To prove (x), we pass to the limit as $\sigma \rightarrow 0$ in the first equation of $\left(\mathbf{P}_{\delta \sigma}\right)$, obtaining

$$
\int_{0}^{T}\left\langle u_{\delta t}, \phi\right\rangle \mathrm{d} t=-\iint_{\Omega_{T}} Q_{\delta}^{1 / 2}\left(u_{\delta}, v_{\delta}\right) w_{\delta} \phi_{x} \quad \forall \phi \in L^{2}\left(0, T ; H^{1}(\Omega)\right) ;
$$


passing to the limit in the second equation of $\left(\mathbf{P}_{\delta \sigma}\right)$ we have

$$
\iint_{\Omega_{T}} v_{\delta t} \psi=-\iint_{\Omega_{T}} Q_{\delta}\left(u_{\delta}, v_{\delta}\right)\left(\mathcal{F}_{v}\left(u_{\delta}, v_{\delta}\right)-\varepsilon v_{\delta x x}\right) \psi \quad \forall \psi \in L^{2}\left(\Omega_{T}\right) .
$$

To identify $w_{\delta}$, we observe that for all $\sigma>0$ and all $\phi \in L^{2}\left(0, T ; H_{0}^{1}(\Omega)\right)$ the following identity holds:

$$
\iint_{\Omega_{T}} Q_{\delta}^{1 / 2}\left(u_{\delta \sigma}, v_{\delta \sigma}\right)\left(\mathcal{F}_{\sigma u}-\varepsilon u_{\delta \sigma x x}\right)_{x} \phi=-\iint_{\Omega_{T}}\left(\mathcal{F}_{\sigma u}\left(u_{\delta \sigma}, v_{\delta \sigma}\right)-\varepsilon u_{\delta \sigma x x}\right)\left(Q_{\delta}^{1 / 2}\left(u_{\delta \sigma}, v_{\delta \sigma}\right) \phi\right)_{x} .
$$

By (3.15), (3.16) and (3.17) we can pass to the limit on the right hand side of (3.18), provided $\phi$ is compactly supported in $\mathcal{D}\left(u_{\delta}\right)$.

\subsection{The degenerate system}

Proposition 3.5 allows us to select a subsequence, which we still denote by $\left(u_{\delta}, v_{\delta}, w_{\delta}\right)$, such that

$$
\begin{aligned}
& u_{\delta}, v_{\delta} \rightarrow u, v \text { uniformly in } \bar{\Omega}_{T}, \\
& u \in C^{0, \frac{1}{2}, \frac{1}{8}}\left(\bar{\Omega}_{T}\right), \quad v \in C^{0, \frac{1}{2}, \frac{1}{4}}\left(\bar{\Omega}_{T}\right) \text {, } \\
& w_{\delta} \longrightarrow w \text { in } L^{2}\left(\Omega_{T}\right) \text {. }
\end{aligned}
$$

We already know by Lemma 3.3 that $(u, v) \in \bar{B}$; in the following Lemma we prove an estimate which shall enable us to improve these bounds. Let $\chi_{A}$ denote the characteristic function of the set $A$, and let

$$
G(u, v)=v[\ln (u-v)+\ln (1-(u+v))] \chi_{v \geqslant 0}-v[\ln (u+v)+\ln (1-(u-v))] \chi_{v \leqslant 0} .
$$

LEMMA 3.6 For all $0<\delta<\delta_{0}, G\left(u_{\delta}, v_{\delta}\right) \in L^{2}\left(\Omega_{T}\right)$, and for $\beta \in(1,4]$ there exist two positive constants $\beta_{0}, C_{4}$ independent of $\delta$ such that for any $t \in(0, T]$

$$
\begin{aligned}
& \frac{1}{\beta-1} \int_{\Omega} Q_{2 \delta}^{1-\beta}\left(v_{\delta}(t)\right)-2 \iint_{\Omega_{t}} Q_{1 \delta}\left(u_{\delta}\right) Q_{2 \delta}^{1-\beta}\left(v_{\delta}\right) G\left(u_{\delta}, v_{\delta}\right)+ \\
& \quad+\varepsilon \beta_{0} \iint_{\Omega_{t}} Q_{1 \delta}\left(u_{\delta}\right) Q_{2 \delta}^{-\beta}\left(v_{\delta}\right)\left(v_{\delta x}\right)^{2} \leqslant \frac{1}{\beta-1} \int_{\Omega} Q_{2 \delta}^{1-\beta}\left(v_{0}\right)+C_{4}+C_{4} \iint_{\Omega_{t}} Q_{2 \delta}^{1-\beta}\left(v_{\delta}\right) .
\end{aligned}
$$

Proof. We choose $\psi=-Q_{2 \delta}\left(v_{\delta}\right)^{-\beta} Q_{2 \delta}^{\prime}\left(v_{\delta}\right) \chi_{[0, t]}$ as a test function in (3.12). Observe that, since $\left(u_{\delta}, v_{\delta}\right) \in \bar{B}$, we have

$$
Q_{1 \delta}^{\prime}\left(u_{\delta}\right)=1-2 u_{\delta}, \quad Q_{2 \delta}^{\prime}\left(v_{\delta}\right)=-2 v_{\delta} .
$$

Since $v_{\delta t} \in L^{2}\left(\Omega_{T}\right)$, for all $t \in(0, T]$ we obtain

$$
\begin{gathered}
\frac{1}{\beta-1} \int_{\Omega} Q_{2 \delta}^{1-\beta}\left(v_{\delta}(t)\right)-\frac{1}{\beta-1} \int_{\Omega} Q_{2 \delta}^{1-\beta}\left(v_{0}\right)=-\iint_{\Omega_{t}} Q_{2 \delta}^{-\beta} Q_{2 \delta}^{\prime} v_{\delta t} \\
=-2 \iint_{\Omega_{t}} Q_{1 \delta} Q_{2 \delta}^{1-\beta} v_{\delta} \mathcal{F}_{v}+2 \varepsilon \iint_{\Omega_{t}} Q_{1 \delta} Q_{2 \delta}^{1-\beta} v_{\delta} v_{\delta x x} .
\end{gathered}
$$


From the definition of $G$ and $\mathcal{F}_{v}$ we can write the identity

$$
\begin{gathered}
G\left(u_{\delta}, v_{\delta}\right)=-v_{\delta} \mathcal{F}_{v}\left(u_{\delta}, v_{\delta}\right)+v_{\delta} \ln \left(u_{\delta}+v_{\delta}\right) \chi_{v_{\delta}>0}-v_{\delta} \ln \left(u_{\delta}-v_{\delta}\right) \chi_{v_{\delta}<0} \\
-v_{\delta} \ln \left(1-\left(u_{\delta}+v_{\delta}\right)\right) \chi_{v_{\delta}<0}+v_{\delta} \ln \left(1-\left(u_{\delta}-v_{\delta}\right)\right) \chi_{v_{\delta}>0}+v_{\delta} V^{\prime}\left(v_{\delta}\right) .
\end{gathered}
$$

Since $u_{\delta} \geqslant 0$, we get

$$
0 \geqslant v_{\delta} \ln \left(u_{\delta}+v_{\delta}\right) \chi_{v_{\delta}>0} \geqslant v_{\delta} \ln \left(v_{\delta}\right) \chi_{v_{\delta}>0} \geqslant-1 / e,
$$

and similar inequalities hold for the other logarithmic terms on the right hand side. Hence

$$
0 \geqslant G\left(u_{\delta}, v_{\delta}\right) \geqslant-\frac{4}{e}-v_{\delta} \mathcal{F}_{v}\left(u_{\delta}, v_{\delta}\right)+v_{\delta} V^{\prime}\left(v_{\delta}\right)
$$

which implies that $G\left(u_{\delta}, v_{\delta}\right) \in L^{2}\left(\Omega_{T}\right)$. Substituting (3.22) into (3.21), we obtain

$$
\begin{aligned}
& \frac{1}{\beta-1} \int_{\Omega} Q_{2 \delta}^{1-\beta}\left(v_{\delta}(t)\right)-\frac{1}{\beta-1} \int_{\Omega} Q_{2 \delta}^{1-\beta}\left(v_{0}\right) \leqslant \\
& \quad \leqslant 2 \iint_{\Omega_{t}} Q_{1 \delta} Q_{2 \delta}^{1-\beta} G+c_{21} \iint_{\Omega_{t}} Q_{2 \delta}^{1-\beta}+2 \varepsilon \iint_{\Omega_{t}} Q_{1 \delta} Q_{2 \delta}^{1-\beta} v_{\delta} v_{\delta x x} .
\end{aligned}
$$

It remains to estimate the last integral at the right hand side of (3.23). Integrating by parts, we write

$$
\begin{aligned}
\iint_{\Omega_{t}} Q_{1 \delta} Q_{2 \delta}^{1-\beta} v_{\delta} v_{\delta x x} & =-\iint_{\Omega_{t}} Q_{1 \delta}^{\prime} Q_{2 \delta}^{1-\beta} v_{\delta} u_{\delta x} v_{\delta x} \\
& -\iint_{\Omega_{t}} Q_{1 \delta} Q_{2 \delta}^{-\beta}\left[Q_{2 \delta}+2(\beta-1) v_{\delta}^{2}\right]\left(v_{\delta x}\right)^{2}=: I_{1}+I_{2} .
\end{aligned}
$$

A straightforward calculation shows that

$$
Q_{2}(v)+2(\beta-1) v^{2} \geqslant \beta_{0}:=\min \left\{\frac{\beta-1}{2}, \frac{1}{4}\right\}>0, \quad-\frac{1}{2} \leqslant v \leqslant \frac{1}{2} .
$$

Since, by its definition, $Q_{2 \delta}(v) \geqslant Q_{2}(v)$ for $v \in[-1 / 2,1 / 2]$, it follows therefore that

$$
I_{2} \leqslant-\beta_{0} \iint_{\Omega_{t}} Q_{1 \delta} Q_{2 \delta}^{-\beta}\left(v_{\delta x}\right)^{2} .
$$

Applying Cauchy-Schwarz and Young's inequalities to $I_{1}$, we have

$$
\left|I_{1}\right| \leqslant \frac{1}{2} \beta_{0} \iint_{\Omega_{t}} Q_{1 \delta} Q_{2 \delta}^{-\beta}\left(v_{\delta x}\right)^{2}+c_{22} \iint_{\Omega_{t}} Q_{1 \delta}^{-1}\left(Q_{1 \delta}^{\prime}\right)^{2} Q_{2 \delta}^{2-\beta} v_{\delta}^{2}\left(u_{\delta x}\right)^{2} .
$$

Since $\left(u_{\delta}, v_{\delta}\right) \in \bar{B}$, we can make use of the following simple properties of $B$ :

$$
(u, v) \in \bar{B} \Longrightarrow\left\{\begin{array}{l}
|v| \leqslant 2 u(1-u) \\
|1-2 u| \leqslant 4\left(\frac{1}{4}-v^{2}\right)
\end{array}\right.
$$


which imply that

$$
\begin{gathered}
\left|v_{\delta}\right| \leqslant 2 u_{\delta}\left(1-u_{\delta}\right) \leqslant 2 Q_{1 \delta}, \\
\left(Q_{1 \delta}^{\prime}\right)^{2}=\left(1-2 u_{\delta}\right)^{2} \leqslant 16\left(\frac{1}{4}-v_{\delta}^{2}\right)^{2} \leqslant 16 Q_{2 \delta}^{2} .
\end{gathered}
$$

Therefore

$$
\left|I_{1}\right| \leqslant \frac{1}{2} \beta_{0} \iint_{\Omega_{t}} Q_{1 \delta} Q_{2 \delta}^{-\beta}\left(v_{\delta x}\right)^{2}+c_{23} \iint_{\Omega_{t}}\left|v_{\delta}\right| Q_{2 \delta}^{4-\beta}\left(u_{\delta x}\right)^{2} \leqslant \frac{1}{2} \beta_{0} \iint_{\Omega_{t}} Q_{1 \delta} Q_{2 \delta}^{-\beta}\left(v_{\delta x}\right)^{2}+c_{24}
$$

since $\beta \leqslant 4$. Collecting the estimates of $I_{1}$ and $I_{2}$, we finally obtain from (3.23)

$$
\begin{aligned}
& \frac{1}{\beta-1} \int_{\Omega} Q_{2 \delta}^{1-\beta}\left(v_{\delta}(t)\right)-\frac{1}{\beta-1} \int_{\Omega} Q_{2 \delta}^{1-\beta}\left(v_{0}\right) \\
& \leqslant c_{25}+2 \iint_{\Omega_{t}} Q_{1 \delta} Q_{2 \delta}^{1-\beta} G+c_{21} \iint_{\Omega_{t}} Q_{2 \delta}^{1-\beta}-\varepsilon \beta_{0} \iint_{\Omega_{t}} Q_{1 \delta} Q_{2 \delta}^{-\beta}\left(v_{\delta x}\right)^{2},
\end{aligned}
$$

which completes the proof of the Lemma.

COROLLARY 3.7 There exists a constant $d>0$ such that

$$
v(x, t) \in\left[-\frac{1}{2}+d, \frac{1}{2}-d\right] \quad \forall(x, t) \in \bar{\Omega}_{T} ;
$$

in addition,

$$
|\mathcal{D}(u) \backslash \mathcal{B}(u, v)|=0 .
$$

Proof. Applying a Gronwall argument to (3.20) with $\beta=3$ and noting that $G\left(u_{\delta}, v_{\delta}\right) \leqslant 0$, we obtain

$$
\int_{\Omega} Q_{2 \delta}^{-2}\left(v_{\delta}(t)\right)-\iint_{\Omega_{t}} Q_{1 \delta}\left(u_{\delta}\right) Q_{2 \delta}^{-2}\left(u_{\delta}\right) G\left(u_{\delta}, v_{\delta}\right)+\iint_{\Omega_{t}} Q_{1 \delta}\left(u_{\delta}\right) Q_{2 \delta}^{-3}\left(v_{\delta}\right)\left(v_{\delta x}\right)^{2} \leqslant c_{26} .
$$

Suppose by contradiction that $v\left(x_{0}, t_{0}\right)=-\frac{1}{2}$. The uniform convergence of $v_{\delta}$ implies that for any $\gamma>0$ there exists $\delta_{\gamma}>0$ such that

$$
\left|v_{\delta}\left(x, t_{0}\right)+\frac{1}{2}\right| \leqslant\left|v_{\delta}\left(x, t_{0}\right)-v\left(x, t_{0}\right)\right|+\left|v\left(x, t_{0}\right)+\frac{1}{2}\right| \leqslant \gamma+C_{1}\left|x-x_{0}\right|^{1 / 2}
$$

for all $\delta<\delta_{\gamma}, x \in \bar{\Omega}$. Therefore, by (3.26) and (3.22)

$$
c_{26} \geqslant \int_{\Omega}\left(\left(\frac{1}{2}+\delta\right)^{2}-v_{\delta}^{2}\right)^{-2} \geqslant \int_{\Omega}(1+\delta)^{-2}\left(\gamma+C_{1}\left|x-x_{0}\right|^{1 / 2}+\delta\right)^{-2} \quad \forall \delta<\delta_{\gamma},
$$

which implies

$$
c_{26} \geqslant \int_{\Omega}\left(\gamma+C_{1}\left|x-x_{0}\right|^{1 / 2}\right)^{-2}
$$


a contradiction for $\gamma$ sufficiently small. Thus $v>-\frac{1}{2}$ in $\bar{\Omega}_{T}$. Similarly we may show that $v<\frac{1}{2}$ in $\bar{\Omega}_{T}$, and the first assertion follows.

To prove the second statement, suppose by contradiction that the set $\mathcal{D}(u) \backslash \mathcal{B}(u, v)$ has positive measure. Without loss of generality we can assume that the set

$$
A=\left\{(x, t) \in \Omega_{T}: u+v=0, u>0\right\}
$$

has positive measure. Then, defining $A^{\prime}=A \cap \mathcal{D}_{\eta}(u)$, we have $\left|A^{\prime}\right| \geqslant|A| / 2$ for $\eta$ sufficiently small. By (3.19) and Lemma 3.3 it follows that

$$
\forall \gamma>0 \exists \delta_{\gamma}>0 \text { s.t. } 0<u_{\delta}+v_{\delta}<\gamma \text { and }\left|u_{\delta}-u\right|<\gamma \text { a.e. in } A^{\prime}, \quad \forall \delta<\delta_{\gamma} ;
$$

it is now easy to check that almost everywhere in $A^{\prime}$

$$
\frac{2 \eta}{3}<u_{\delta}<\frac{1}{2}+\frac{\eta}{3} \quad \text { and } \quad v_{\delta}<-\frac{\eta}{3} \quad \forall \gamma \in\left(0, \frac{\eta}{3}\right), \delta<\delta_{\gamma} .
$$

From (3.26) and the definition of $G$, we obtain

$$
\iint_{\Omega_{T}} Q_{1 \delta} Q_{2 \delta}^{-2}\left|v_{\delta} \ln \left(u_{\delta}+v_{\delta}\right)\right| \chi_{v_{\delta}} \leqslant 0 \leqslant-\iint_{\Omega_{T}} Q_{1 \delta} Q_{2 \delta}^{-2} G\left(u_{\delta}, v_{\delta}\right) \leqslant c_{26} .
$$

Since

$$
\left|v_{\delta} \ln \left(u_{\delta}+v_{\delta}\right) \chi_{v_{\delta}>0}\right| \leqslant\left|v_{\delta} \ln \left(v_{\delta}\right) \chi_{v_{\delta}>0}\right| \leqslant \frac{1}{e},
$$

using (3.29) and (3.25) we conclude that

$$
\iint_{\Omega_{T}} Q_{1 \delta}\left|v_{\delta} \ln \left(u_{\delta}+v_{\delta}\right)\right| \leqslant c_{27},
$$

and a contradiction now follows for $\gamma$ sufficiently small, taking (3.28) into account and arguing as in the proof of Lemma 3.3.

In order to pass to the limit as $\delta \rightarrow 0$ we need an estimate independent of $\delta$ on the second derivatives of $u_{\delta}, v_{\delta}$ and for the singular terms $\mathcal{F}_{u}\left(u_{\delta}, v_{\delta}\right), \mathcal{F}_{v}\left(u_{\delta}, v_{\delta}\right)$. The next Lemma provides such an estimate on any compact subset of $\mathcal{D}(u)$.

LEMma 3.8 For any $K \subset \mathcal{D}(u)$ compact there exist positive constants $\delta_{K}$ and $C_{K}$ such that the following estimates hold for all $\delta<\delta_{K}$ :

$$
\begin{array}{ll}
\left\|u_{\delta x x}\right\|_{L^{2}(K)} \leqslant C_{K}, & \left\|\mathcal{F}_{u}\left(u_{\delta}, v_{\delta}\right)\right\|_{L^{2}(K)} \leqslant C_{K}, \\
\left\|v_{\delta x x}\right\|_{L^{2}(K)} \leqslant C_{K}, & \left\|\mathcal{F}_{v}\left(u_{\delta}, v_{\delta}\right)\right\|_{L^{2}(K)} \leqslant C_{K} .
\end{array}
$$

Proof. Let $K \subset \mathcal{D}(u)$ compact; then $K \subseteq \mathcal{D}_{\eta}(u)$ for some $\eta>0$. Since there exists a positive distance between $\mathcal{D}_{\eta}(u)$ and $\bar{\Omega}_{T} \backslash \mathcal{D}_{\eta / 2}(u)$, it is possible to select $\zeta \in C^{\infty}\left(\bar{\Omega}_{T}\right)$ such that $0 \leqslant \zeta \leqslant 1$, $\zeta=1$ in $\mathcal{D}_{\eta}(u), \zeta=0$ in $\bar{\Omega}_{T} \backslash \mathcal{D}_{\eta / 2}(u)$ and $\zeta_{x}=0$ over $S_{T}$.

Choosing $\psi=\left[Q_{\delta}\left(u_{\delta \sigma}, v_{\delta \sigma}\right)\right]^{-1} \zeta^{2} v_{\delta \sigma x x}$ as a test function in the second equation in $\left(\mathbf{P}_{\delta \sigma}\right)$, we have

$$
\iint_{\Omega_{T}} \zeta^{2} Q_{\delta}^{-1} v_{\delta \sigma x x} v_{\delta \sigma t}=-\iint_{\Omega_{T}} \zeta^{2}\left(\mathcal{F}_{\sigma v}-\varepsilon v_{\delta \sigma x x}\right) v_{\delta \sigma x x} .
$$


By the choice of $\zeta$, Corollary 3.7 and the equicontinuity of $\left\{u_{\delta \sigma}\right\}$ and $\left\{v_{\delta \sigma}\right\}$, the following properties hold for $0<\delta<\delta_{K}$ and $0<\sigma<\sigma(\delta)$ :

$$
\begin{gathered}
u_{\delta \sigma} \in\left[\frac{\eta}{4}, 1-\frac{\eta}{4}\right] \text { in } \operatorname{supp} \zeta, \\
v_{\delta \sigma} \in\left[-\frac{1}{2}+\frac{d}{4}, \frac{1}{2}-\frac{d}{4}\right] .
\end{gathered}
$$

Hence

$$
\left|\iint_{\Omega_{T}} \zeta^{2} Q_{\delta}^{-1} v_{\delta \sigma x x} v_{\delta \sigma t}\right| \leqslant \frac{1}{2 \varepsilon} Q_{1 \delta}^{-1}\left(\frac{\eta}{4}\right) Q_{2 \delta}^{-1}\left(\frac{1}{2}-\frac{d}{4}\right) \iint_{\Omega_{T}} \zeta^{2} Q_{\delta}^{-1}\left(v_{\delta \sigma t}\right)^{2}+\frac{\varepsilon}{2} \iint_{\Omega_{T}} \zeta^{2}\left(v_{\delta \sigma x x}\right)^{2},
$$

where we have used the Cauchy-Schwarz and Young's inequalities. Therefore, it follows from (3.30) and Lemma 3.1 (vi) that

$$
\frac{\varepsilon}{2} \iint_{\Omega_{T}} \zeta^{2}\left(v_{\delta \sigma x x}\right)^{2}-\iint_{\Omega_{T}} \zeta^{2} \mathcal{F}_{\sigma v} v_{\delta \sigma x x} \leqslant c_{28} \quad \forall \delta<\delta_{K}, \sigma<\sigma(\delta) .
$$

We now choose $\phi=\zeta^{2} \tilde{Q}_{1 \delta}\left(u_{\delta \sigma}\right)\left[Q_{2 \delta}\left(v_{\delta \sigma}\right)\right]^{-1}$ as a test function in the first equation of $\left(\mathbf{P}_{\delta \sigma}\right)$, where $\tilde{Q}_{1 \delta}(u)$ is defined by

$$
\tilde{Q}_{1 \delta}(u)=\int_{1 / 2}^{u} \frac{1}{Q_{1 \delta}(s)} \mathrm{d} s
$$

obtaining

$$
\begin{aligned}
\int_{0}^{T}\left\langle u_{\delta \sigma t}, \zeta^{2} \tilde{Q}_{1 \delta} Q_{2 \delta}^{-1}\right\rangle & \mathrm{d} t=-\iint_{\Omega_{T}} \zeta^{2} u_{\delta \sigma x}\left(\mathcal{F}_{\sigma u}-\varepsilon u_{\delta \sigma x x}\right)_{x} \\
& +\iint_{\Omega_{T}} \zeta^{2} Q_{1 \delta} \tilde{Q}_{1 \delta} Q_{2 \delta}^{-1} Q_{2 \delta}^{\prime} v_{\delta \sigma x}\left(\mathcal{F}_{\sigma u}-\varepsilon u_{\delta \sigma x x}\right)_{x} \\
& -\iint_{\Omega_{T}}\left(\zeta^{2}\right)_{x} Q_{1 \delta} \tilde{Q}_{1 \delta}\left(\mathcal{F}_{\sigma u}-\varepsilon u_{\delta \sigma x x}\right)_{x}=: I_{1}+I_{2}+I_{3} .
\end{aligned}
$$

It easily follows from the energy estimates in Lemma 3.1 and the definition of $\zeta$ that

$$
\left|\int_{0}^{T}\left\langle u_{\delta \sigma t}, \zeta^{2} \tilde{Q}_{1 \delta} Q_{2 \delta}^{-1}\right\rangle \mathrm{d} t\right|+\left|I_{2}\right|+\left|I_{3}\right| \leqslant c_{29} \quad \forall \delta<\delta_{K}, \sigma<\sigma(\delta) .
$$

Let $\gamma>0$ be a constant to be chosen later: integrating $I_{1}$ by parts and using the Cauchy-Schwarz and Young's inequalities we find that

$$
I_{1} \leqslant \iint_{\Omega_{T}} \zeta^{2} \mathcal{F}_{\sigma u} u_{\delta \sigma x x}-\frac{\varepsilon}{2} \iint_{\Omega_{T}} \zeta^{2}\left(u_{\delta \sigma x x}\right)^{2}+\frac{\gamma}{2} \iint_{\Omega_{T}} \zeta^{2}\left(\mathcal{F}_{\sigma u}\right)^{2}+2\left(\varepsilon+\frac{1}{\gamma}\right) \iint_{\Omega_{T}}\left(\zeta_{x}\right)^{2}\left(u_{\delta \sigma x}\right)^{2} .
$$


Note that the last integral on the right hand side is uniformly bounded. Because of the definition of $\zeta$ and arguing as in the proof of Proposition 3.5, we find that

$$
\frac{\gamma}{2} \iint_{\Omega_{T}} \zeta^{2}\left(\mathcal{F}_{\sigma u}\right)^{2} \leqslant c_{30}+4 \gamma \iiint_{\Omega_{T}} \zeta^{2}\left(\mathcal{F}_{\sigma v}\right)^{2}
$$

We now proceed to bound the right hand side of (3.33):

$$
\begin{gathered}
4 \gamma \iint_{\Omega_{T}} \zeta^{2}\left(\mathcal{F}_{\sigma v}\right)^{2} \leqslant 8 \gamma \iint_{\Omega_{T}} \zeta^{2}\left(\mathcal{F}_{\sigma v}-\varepsilon v_{\delta \sigma x x}\right)^{2}+8 \gamma \varepsilon^{2} \iint_{\Omega_{T}} \zeta^{2}\left(v_{\delta \sigma x x}\right)^{2} \\
\leqslant 8 \gamma c_{31} \iint_{\Omega_{T}} \zeta^{2} Q_{\delta}\left(\mathcal{F}_{\sigma v}-\varepsilon v_{\delta \sigma x x}\right)^{2}+8 \gamma \varepsilon^{2} \iint_{\Omega_{T}} \zeta^{2}\left(v_{\delta \sigma x x}\right)^{2}
\end{gathered}
$$

and therefore, choosing $\gamma<1 /(32 \varepsilon)$ and recalling Lemma 3.1 (iv) it turns out that

$$
4 \gamma \iint_{\Omega_{T}} \zeta^{2}\left(\mathcal{F}_{\sigma v}\right)^{2} \leqslant c_{32}+\frac{\varepsilon}{4} \iint_{\Omega_{T}} \zeta^{2}\left(v_{\delta \sigma x x}\right)^{2}
$$

Summing (3.31) and (3.32) and using the estimates above we have

$$
\frac{\varepsilon}{2} \iint_{\Omega_{T}} \zeta^{2}\left(u_{\delta \sigma x x}\right)^{2}+\frac{\varepsilon}{4} \iint_{\Omega_{T}} \zeta^{2}\left(v_{\delta \sigma x x}\right)^{2}-\iint_{\Omega_{T}} \zeta^{2}\left[\mathcal{F}_{\sigma u} u_{\delta \sigma x x}+\mathcal{F}_{\sigma v} v_{\delta \sigma x x}\right] \leqslant c_{33} .
$$

Integrating by parts the last integral at the left hand side and recalling the identity (3.10), we obtain

$$
\begin{aligned}
-\iint_{\Omega_{T}} \zeta^{2}\left[\mathcal{F}_{\sigma u} u_{\delta \sigma x x}+\mathcal{F}_{\sigma v} v_{\delta \sigma x x}\right]= \\
\quad=-\iint_{\Omega_{T}}\left(\zeta^{2}\right)_{x x} \mathcal{F}_{\sigma}+\iint_{\Omega_{T}} \zeta^{2}\left[U^{\prime \prime}\left(u_{\delta \sigma}\right)\left(u_{\delta \sigma x}\right)^{2}+V^{\prime \prime}\left(v_{\delta \sigma}\right)\left(v_{\delta \sigma x}\right)^{2}\right] \\
\quad+\iint_{\Omega_{T}} \zeta^{2}\left[\varphi_{\sigma}\left(u_{\delta \sigma}+v_{\delta \sigma}\right)\left[\left(u_{\delta \sigma}+v_{\delta \sigma}\right)_{x}\right]^{2}+\varphi_{\sigma}\left(u_{\delta \sigma}-v_{\delta \sigma}\right)\left[\left(u_{\delta \sigma}-v_{\delta \sigma}\right)_{x}\right]^{2}\right] .
\end{aligned}
$$

Since $\mathcal{F}_{\sigma}$ is uniformly bounded in $L^{1}\left(\Omega_{T}\right)$ by Lemma 3.1 (vii), the second integral is uniformly bounded and the third is positive, we obtain

$$
\iint_{\Omega_{T}} \zeta^{2}\left[\mathcal{F}_{\sigma u} u_{\delta \sigma x x}+\mathcal{F}_{\sigma v} v_{\delta \sigma x x}\right] \leqslant c_{34}
$$

Therefore, from (3.35), we can conclude that

$$
\frac{\varepsilon}{2} \iint_{\Omega_{T}} \zeta^{2}\left(u_{\delta \sigma x x}\right)^{2}+\frac{\varepsilon}{4} \iint_{\Omega_{T}} \zeta^{2}\left(v_{\delta \sigma x x}\right)^{2} \leqslant c_{35} \quad \forall \delta<\delta_{K}, \sigma<\sigma(\delta) .
$$


Combining this estimate with (3.34) and (3.33) we obtain, moreover, that

$$
\iint_{\Omega_{T}} \zeta^{2}\left(\mathcal{F}_{\sigma u}\right)^{2} \leqslant c_{36}, \text { and } \iint_{\Omega_{T}} \zeta^{2}\left(\mathcal{F}_{\sigma v}\right)^{2} \leqslant c_{37} \text {. }
$$

The assertion now follows by means of the definition of $\zeta$, the lower semicontinuity of $L^{2}$-norms and the arbitrariness of $K$.

Proof of Theorem 1. Let $\left(u_{\delta}, v_{\delta}\right)$ be the sequence given in (3.19). Using Proposition 3.5 and Lemma 3.8 we have

$$
\begin{array}{rllll}
u_{\delta}, v_{\delta} & -* & u, v & \text { in } & L^{\infty}\left(0, T ; H^{1}(\Omega)\right), \\
u_{\delta x x}, v_{\delta x x} & \longrightarrow & u_{x x}, v_{x x} & \text { in } & L_{\mathrm{loc}}^{2}(\mathcal{D}(u)), \\
u_{\delta t} & \longrightarrow & u_{t} & \text { in } & L^{2}\left(0, T ;\left(H^{1}(\Omega)\right)^{\prime}\right), \\
v_{\delta t} & \longrightarrow & v_{t} & \text { in } & L^{2}\left(\Omega_{T}\right),
\end{array}
$$

and (i)-(iii) follow. Property (iv) is a consequence of Corollary 3.7. From Lemma 3.8 we obtain

$$
\begin{aligned}
& \mathcal{F}_{u}\left(u_{\delta}, v_{\delta}\right) \longrightarrow g_{1} \text { in } L_{\text {loc }}^{2}(\mathcal{D}(u)), \\
& \mathcal{F}_{v}\left(u_{\delta}, v_{\delta}\right) \longrightarrow g_{2} \text { in } L_{\text {loc }}^{2}(\mathcal{D}(u)) .
\end{aligned}
$$

Since $|\mathcal{D}(u) \backslash \mathcal{B}(u, v)|=0$ and $u_{\delta}, v_{\delta} \rightarrow u, v$ uniformly in $\bar{\Omega}_{T}$, we have

$$
\mathcal{F}_{u}\left(u_{\delta}, v_{\delta}\right) \longrightarrow \mathcal{F}_{u}(u, v) \text { a.e. in } \mathcal{D}(u),
$$

and therefore $g_{1}=\mathcal{F}_{u}(u, v)$. By the same argument $g_{2}=\mathcal{F}_{v}(u, v)$ and $(\mathrm{v})$ is proved.

Property (vi) is straightforward. To prove (vii), let $K \subset \mathcal{D}(u)$ compact; then $K \subseteq \mathcal{D}_{\eta}(u)$ for some $\eta>0$. Since $u \in C\left(\bar{\Omega}_{T}\right)$, we can select $\zeta \in C^{\infty}\left(\bar{\Omega}_{T}\right)$ such that $0 \leqslant \zeta \leqslant 1, \zeta=1$ in $\mathcal{D}_{\eta}(u)$ and $\zeta=0$ in $\bar{\Omega}_{T} \backslash \mathcal{D}_{\eta / 2}(u)$. Hence we have

$$
\begin{gathered}
\left\|\zeta u_{\delta}\right\|_{L^{2}\left(0, T ; H^{2}(\Omega)\right)} \leqslant c_{38}, \\
\left\|\left(\zeta u_{\delta}\right)_{t}\right\|_{L^{2}\left(0, T ;\left(H^{1}(\Omega)\right)^{\prime}\right)} \leqslant c_{39},
\end{gathered}
$$

which implies that

$$
\left(\zeta u_{\delta}\right)_{x} \longrightarrow(\zeta u)_{x} \text { in } L^{2}\left(0, T ; H^{1-\lambda}(\Omega)\right), \quad \lambda>0 .
$$

The following continuous embedding and linear mapping

$$
L^{2}\left(0, T ; H^{1-\lambda}(\Omega)\right) \rightarrow L^{2}\left(0, T ; H^{\frac{1}{2}-\lambda}(\partial \Omega)\right) \subset L^{2}\left(0, T ; L^{2}(\partial \Omega)\right), \quad \lambda<\frac{1}{2},
$$

yield

$$
\left.\left.\left(\zeta u_{\delta}\right)_{x}\right|_{S_{T}} \rightarrow(\zeta u)_{x}\right|_{S_{T}} \text { in } L^{2}\left(S_{T}\right)
$$

and therefore $\left.u_{x}\right|_{S_{T} \cap K}=0$. Now (vii) follows applying the same argument to $v_{x}$.

Finally we pass to the limit as $\delta \rightarrow 0$ in equations (3.11)-(3.13) to prove (viii). The limit is straightforward in equations (3.11) and (3.12). With respect to (3.13) we observe that

$$
\left(Q_{\delta}^{1 / 2} \phi\right)_{x}=\frac{1}{2} Q_{1 \delta}^{-1 / 2} Q_{1 \delta}^{\prime} Q_{2 \delta}^{1 / 2} u_{\delta x} \phi+\frac{1}{2} Q_{2 \delta}^{-1 / 2} Q_{2 \delta}^{\prime} Q_{1 \delta}^{1 / 2} v_{\delta x} \phi+Q_{\delta}^{1 / 2} \phi_{x}
$$


and, for all $\phi \in L^{2}\left(0, T ; H_{0}^{1}(\Omega)\right)$ with compact support in $\mathcal{D}(u)$, each term converges strongly in $L^{2}\left(\Omega_{T}\right)$ because of (3.36) (with the analogous convergence for $v$ ) and the fact that $v \in\left(-\frac{1}{2}, \frac{1}{2}\right)$. This completes the proof of the Theorem.

\section{Conclusions}

Further questions regarding existence might focus around extending our results to higher space dimensions, as well as, attaining control on the measure and perhaps boundary of the set on which $u=0$ or 1 .

We remark that our analysis does not depend heavily on the choice of the polynomial terms in the free energy; in particular, an appropriate third or fourth order polynomial could be taken to model the behaviour of the free energy in the vicinity of the triple point as in [15].

With regard to limiting motion for the Allen-Cahn/Cahn-Hilliard system (P), it would be nice to be able to justify the predictions of the formal asymptotic analysis. But, as the asymptotic analysis has yet to be justified even for the case of a single Cahn-Hilliard equation with degenerate mobility and logarithmic free energy, such a task is at present somewhat remote. However, our anaylsis provides a step in this direction, and later on, hopefully, numerical schemes can be developed.

Finally, it would be nice to be able to treat other systems which couple degenerate fourth and second order equations which have a less distinct structure.

\section{Acknowledgements}

One of the authors (A. N.-C.) would like to acknowledge the support and hospitality of the Istituto per le Applicazioni del Calcolo "M. Picone" - CNR - Roma (Italy), as well as the support of the Fund for the Promotion of Research at the Technion. The research of A. Novick-Cohen was partially supported by the National Science Foundation through the Center for Nonlinear Analysis.

\section{REFERENCES}

1. Allen, S. E. \& CAhn, J. W. A microscopic theory for antiphase boundary motion and its application to antiphase domain coarsening. Acta Met. 27, (1979) 1085-1095.

2. Barrett, J. W. \& Blowey, J. F. Finite element approximation of the Cahn-Hilliard equation with concentration dependent mobility. Mathematics of Computation 68, (1999) 487-517.

3. Barrett, J. W., Blowey, J. F., \& Garcke, H. Finite element approximation of the Cahn-Hilliard equation with degenerate mobility. SIAM J. Num. Anal. (to appear).

4. Beretta, E., Bertsch, M., \& Dal Passo, R. Nonnegative solutions of a fourth-order nonlinear degenerate parabolic equation. Arch. Rational Mech. Anal. 129, (1995) 175-200.

5. BERnis, F. \& FriedmAn, A. Higher order nonlinear degenerate parabolic equations. J. Differential Equations 83, (1990) 179-206.

6. Bertsch, M., Dal Passo, R., \& UGhi, M. Discontinuous "viscosity" solutions of a degenerate parabolic equation. Trans. Amer. Math. Soc. 320, (1990) 779-798.

7. Blowey, J. F. \& Elliott, C. M. The Cahn-Hilliard gradient theory for phase separation with nonsmooth free energy - Part I: Mathematical analysis. Euro. J. Appl. Math. 2, (1991) 233-279.

8. Blowey, J. F. \& Elliott, C. M. The Cahn-Hilliard gradient theory for phase separation with nonsmooth free energy - Part II: Numerical analysis. Euro. J. Appl. Math. 3, (1992) 147-179.

9. Brochet, D., Hilhorst, D., \& Novick-Cohen, A. Inertial sets for Cahn-Hilliard/Cahn-Allen systems. Appl. Math. Lett. 7, (1994) 83-87. 
10. Bronsard, L. GarcKe, H., \& STOTh, B. A multi-phase Mullins-Sekerka system: matched asymptotic expansions and an implicit time discretization for the geometric evolution problem. Proc. Roy. Soc. Edinburgh 128 A, (1998) 481-506.

11. BRONSARD, L. \& ReITICH, F. On three-phase boundary motion and the singular limit of a vector-valued Ginzburg-Landau equation. Arch. Rat. Mech. Anal. 124, (1993) 355-379.

12. Cahn, J. W., Elliott, C. M., \& Novick-Cohen, A. The Cahn-Hilliard equation with a concentration dependent mobility: motion by minus the Laplacian of the mean curvature. Euro. J. Appl. Math. 7, (1996) 287-301.

13. CAhn, J. W. \& Novick-Cohen, A. Evolution equations for phase separation and ordering in binary alloys. J. Stat. Phys. 76, (1994) 877-909.

14. Cahn, J. W. \& Novick-Cohen, A. Limiting motion for an Allen-Cahn/Cahn-Hilliard system. In Niezgodka, N. \& Strzelecki, P. (eds), Free Boundary Problems and Applications. Addison Wesley Longman, London (1996).

15. Cahn, J. W. \& Novick-Cohen, A. Motion by curvature and impurity drag: resolution of a paradox, Preprint.

16. Chen, X. Global asymptotic limit of the Cahn-Hilliard equation, Preprint.

17. Dal Passo, R., Garcke H., \& GrÜN, G. On a fourth-order degenerate parabolic equation: global entropy estimates, existence, and qualitative behaviour of solutions. SIAM J. Math. Anal. 29, (1998) 321-342.

18. Dal PAsso, R. \& Luckhaus, S. A degenerate diffusion problem not in divergence form. J. Differential Equations 69, (1987) 1-14.

19. Elliott, C. M. \& Garcke, H. On the Cahn-Hilliard equation with degenerate mobility. SIAM J. Math. Anal. 27, (1996) 404-423.

20. Elliott, C. M. \& GARCKe, H. Diffusional phase transitions in multicomponent systems with a concentration dependent mobility matrix. Physica D 109, (1997) 242-256.

21. Elliott, C. M. \& Luckhaus, S. A generalized diffusion equation for phase separation of a multicomponent mixture with interfacial free energy. IMA, University of Minnesota, Preprint 887 (1991).

22. Evans, L. C., Soner, H. M., \& Souganidis, P. Phase transitions and generalized mean curvature flow equations. Comm. Pure Appl. Math. 45, (1992) 1097-1123.

23. Garcke, H. \& Novick-Cohen, A. A singular limit for a system of degenerate Cahn-Hilliard equations. Advances Diff. Eqns (to appear).

24. Novick-Cohen, A. The Cahn-Hilliard equation: mathematical and modeling perspectives. Adv. Math. Sci. Appl. 8, (1998) 965-985.

25. Novick-Cohen, A. Triple junction motion for Allen-Cahn/Cahn-Hilliard systems. Physica D, (to appear).

26. Novick-Cohen, A. Energy methods for the Cahn-Hilliard equation. Quart. Appl. Math. 46, (1988) 681-690.

27. Pego, R. L. Front migration in the nonlinear Cahn-Hilliard equation. Proc. Roy. Soc. London A 422, (1989) 116-133.

28. Rubinstein, J., Sternberg, P., \& Keller, J. Fast reaction, slow diffusion, and curve shortening. SIAM J. Appl. Math. 49, (1989) 116-133.

29. Simon, J. Compact sets in the space $L^{p}(0, T ; B)$. Annali Mat. Pura Appl. 146, (1987) 65-97. 